Supporting information for:

\title{
Substituent Effect on exo Stereoselectivity in the 1,3-Dipolar Cycloaddition Reaction of Tulipalin A with Nitrile Ylides
}

\author{
Petr Melša, ${ }^{\dagger}$ Michal Čajan, ${ }^{\star}{\text { Zdeněk } \text { Havlas }^{\S} \text { and Ctibor Mazal }}^{\dagger *}$ \\ †'Department of Chemistry, Faculty of Science, Masaryk University, Kotlářská 2, 61137 Brno, \\ Czech Republic

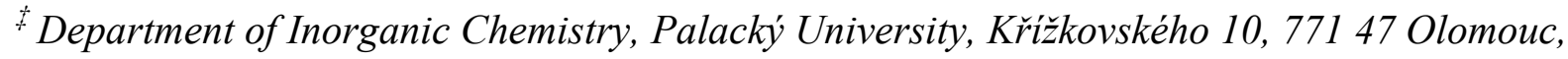 \\ Czech Republic \\ $\S$ Institute of Organic Chemistry and Biochemistry, Academy of Sciences of the Czech Republic, \\ Flemingovo nám. 2, 16610 Prague 6, Czech Republic
}

\section{Supporting Information Table of Contents}

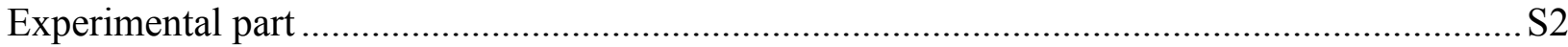

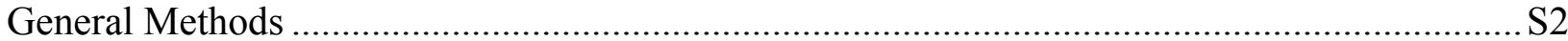

General Procedure for Preparation of 4-Substituted Benzyl Benzamides. ................................. S2

General Procedure for Preparation of Benzimidoyl Chlorides. ..................................................S3

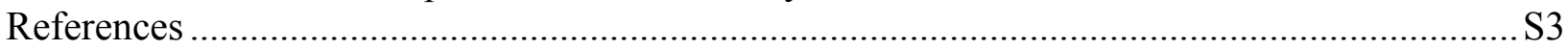

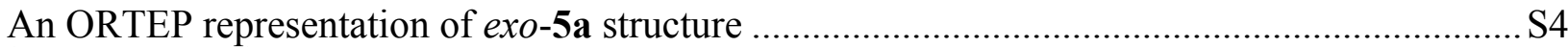

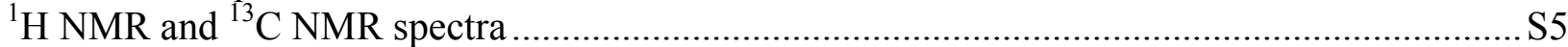

${ }^{1} \mathrm{H}$ NMR Spectra of crude reaction mixtures for exo/endo ratios determination ......................... S18

Hammett-like plots correlating $\log ($ d.e. $)$ with substituent sigma values $\sigma_{p}^{\circ}$ and $\sigma_{m}$............... S26

Cartesian coordinates of optimized transition states ...................................................... S27

$\Delta E$ and $\Delta G$ energies, entropy $(\Delta S)$, and imaginary frequencies $\left(v_{\text {img }}\right)$ of optimized TSs ......... S41 


\section{Experimental Part}

General Methods. Melting points were obtained on Kofler block and are uncorrected. ${ }^{1} \mathrm{H}$ and ${ }^{13} \mathrm{C}$ NMR spectra were obtained on a $300 \mathrm{MHz}$ spectrometer in $\mathrm{CDCl}_{3}$. Chemical shifts are given in ppm $(\delta$-scale $)$, and are corrected to the residual peak of solvent $\left(\delta=7.26 \mathrm{ppm}\right.$ for ${ }^{1} \mathrm{H}$, and $\delta=$ 77.23 ppm for ${ }^{13} \mathrm{C}$ ). IR spectra (wave-numbers in $\mathrm{cm}^{-1}$ ) were measured in $\mathrm{KBr}$ pellets on a FTIR spectrometer. HRMS with an electrospray ionisation in the negative mode was performed using the following parameters: source block/desolvation temperature of $90 / 200{ }^{\circ} \mathrm{C}$, capillary/cone voltage of $2500 / 30 \mathrm{~V}$, and spray/cone gas flow $\left(\mathrm{N}_{2}\right)$ of 50/250 litres/h. In the full-scan mode, data acquisition was performed in the range 50-1000 Da, with a cycle time of $28 \mu \mathrm{s}$, a scan time of $1.0 \mathrm{~s}$ and collision energy of $4 \mathrm{~V}$. For the exact mass determination experiments, a lock spray technology was used as the external calibration with a mixture of $0.1 \mathrm{M} \mathrm{NaOH} / 10 \%$ (v/v) formic acid/acetonitrile (1:1:8 by volume) as a reference. Anions $\left(\mathrm{M}-\mathrm{H}_{2}-1\right)^{-}$were detected in instead of $(\mathrm{M}-1)^{-}$due to an easy 1,4-elimination of hydrogen from $\mathbf{6}$. The accurate masses were calculated and used for the determination of the elementary composition and structure with fidelity 5 ppm and better. Tulipalin A was synthesized according to the published procedure. ${ }^{1}$

General Procedure for Preparation of 4-Substituted Benzyl Benzamides 8a-g. To the mixture of amine $(7 \mathrm{mmol})$, methylene chloride $(30 \mathrm{ml})$ and $\mathrm{Na}_{2} \mathrm{CO}_{3}(14 \mathrm{mmol})$ dissolved in water $(10 \mathrm{ml})$, a slight excess of benzoyl chloride $(7.4 \mathrm{mmol})$ was added dropwise over the period of 23 minutes. The mixture was stirred for approximately 30 minutes and then the organic layer was separated, and the aqueous one was extracted with methylene chloride $(3 \times 20 \mathrm{ml})$. Combined organic portions were filtered through a pad of silica gel and dried over $\mathrm{MgSO}_{4}$. Recrystallization from $50 \%$ aqueous methanol afforded corresponding benzamide.

$N$-Benzylbenzamide (8a). (1.38 g, 93 \%). M.p. $104.2-106.3{ }^{\circ} \mathrm{C} ;{ }^{1} \mathrm{H}$ NMR $\left(300 \mathrm{MHz}, \mathrm{CDCl}_{3}\right) \delta$ $7.79(\mathrm{~d}, J=6.9 \mathrm{~Hz}, 2 \mathrm{H}), 7.53-7.30(\mathrm{~m}, 8 \mathrm{H}), 4.66(\mathrm{~d}, J=5.7 \mathrm{~Hz}, 2 \mathrm{H})\left(c f . \mathrm{ref}^{2}\right)$.

$\boldsymbol{N}$-(4-Nitrobenzyl)benzamide (8b). (1.40 g, 78 \%). M.p. 144.0 - $146.4{ }^{\circ} \mathrm{C} ;{ }^{1} \mathrm{H}$ NMR $(300 \mathrm{MHz}$, $\left.\mathrm{CDCl}_{3}\right) \delta 8.21(\mathrm{~d}, J=8.6 \mathrm{~Hz}, 2 \mathrm{H}), 7.82(\mathrm{~d}, J=7.4 \mathrm{~Hz}, 2 \mathrm{H}), 7.57-7.43(\mathrm{~m}, 3 \mathrm{H}), 7.53(\mathrm{~d}, J=8.6$ $\mathrm{Hz}, 2 \mathrm{H}), 4.77(\mathrm{~d}, J=6.0 \mathrm{~Hz}, 2 \mathrm{H})\left(c f . \mathrm{ref}^{3}\right)$.

$\mathrm{N}$-(4-Methylbenzyl)benzamide (8c). (1.25 g, 79 \%). M.p. $140.7-141.8{ }^{\circ} \mathrm{C} ;{ }^{1} \mathrm{H}$ NMR $(300$ $\left.\mathrm{MHz}, \mathrm{CDCl}_{3}\right) \delta 7.79(\mathrm{~d}, J=7.55 \mathrm{~Hz}, 2 \mathrm{H}), 7.51-7.42(\mathrm{~m}, 3 \mathrm{H}), 7.28-7.17(\mathrm{~m}, 4 \mathrm{H}), 4.63(\mathrm{~d}, J=$ $5.5 \mathrm{~Hz}, 2 \mathrm{H}), 2.37$ (s, 3H) (cf. $\left.\operatorname{ref}^{4}\right)$.

$\boldsymbol{N}$-(4-Phenylbenzyl)benzamide (8d). (1.79 g, 89 \%). M.p. $166.9-168.3{ }^{\circ} \mathrm{C} ;{ }^{1} \mathrm{H}$ NMR $(300$ $\left.\mathrm{MHz} \mathrm{CDCl}_{3}\right) \delta 7.81(\mathrm{~d}, J=6.9 \mathrm{~Hz}, 2 \mathrm{H}), 7.60-7.58(\mathrm{~m}, 4 \mathrm{H}), 7.52-7.35(\mathrm{~m}, 8 \mathrm{H}), 4.71(\mathrm{~d}, J=$ $5.7 \mathrm{~Hz}, 2 \mathrm{H})\left(c f . \mathrm{ref}^{5}\right)$.

$\boldsymbol{N}$-(4-Fluorobenzyl)benzamide (8e). (1.33 g, 83 \%). M.p. $122.6-124.1{ }^{\circ} \mathrm{C} ;{ }^{1} \mathrm{H}$ NMR $(300 \mathrm{MHz}$, $\left.\mathrm{CDCl}_{3}\right) \delta 7.79(\mathrm{~d}, J=6.9 \mathrm{~Hz}, 2 \mathrm{H}), 7.54-7.41(\mathrm{~m}, 2 \mathrm{H}), 7.36-7.31(\mathrm{~m}, 2 \mathrm{H}), 7.04(\mathrm{t}, J=8.7$, $2 \mathrm{H}), 4.62(\mathrm{~d}, J=5.8 \mathrm{~Hz}, 2 \mathrm{H})\left(c f . \mathrm{ref}^{6}\right)$.

$\boldsymbol{N}$-(4-Bromobenzyl)benzamide (8f). (1.70 g, 85 \%). M.p. $143.7-144.5{ }^{\circ} \mathrm{C} ;{ }^{1} \mathrm{H} \mathrm{NMR}(300 \mathrm{MHz}$, $\left.\mathrm{CDCl}_{3}\right) \delta 7.79(\mathrm{~d}, J=7.8 \mathrm{~Hz}, 2 \mathrm{H}), 7.52-7.42(\mathrm{~m}, 4 \mathrm{H}), 7.25(\mathrm{~d}, J=9.93 \mathrm{H}), 4.61(\mathrm{~d}, J=5.6 \mathrm{~Hz}$, $\left.2 \mathrm{H}, \mathrm{CH}_{2}\right)\left(c f . \mathrm{ref}^{3}\right)$.

$\boldsymbol{N}$-(4-Chlorobenzyl)benzamide (8g). (1.31 g, 76 \%). M.p. $139.7-143.0{ }^{\circ} \mathrm{C} ;{ }^{1} \mathrm{H}$ NMR $(300$ $\left.\mathrm{MHz} \mathrm{CDCl}_{3}\right) \delta 7.79(\mathrm{~d}, J=7.0 \mathrm{~Hz}, 2 \mathrm{H}), 7.52-7.42(\mathrm{~m}, 3 \mathrm{H}), 7.34-7.26(\mathrm{~m}, 4 \mathrm{H}), 4.62(\mathrm{~d}=5.8$ $\mathrm{Hz}, 2 \mathrm{H})\left(c f \cdot \mathrm{ref}^{3}\right)$. 
General Procedure for Preparation of Benzimidoyl Chlorides 7a-g. The mixture of amide ( $7 \mathrm{mmol}$ ) and thionyl chloride $(28 \mathrm{mmol})$ was stirred for $4-5 \mathrm{~h}$ under $\mathrm{N}_{2}$ atmosphere at the temperature of $45-50{ }^{\circ} \mathrm{C}$. The excessive thionyl chloride was removed in vacuo, and obtained residue was re-dissolved in dry benzene $(3 \mathrm{ml})$ and concentrated once again to remove remaining thionyl chloride. Resulting imidoyl chloride containing less than $5 \%$ of starting material was used in cycloaddition reactions without further purification.

$\boldsymbol{N}$-Benzylbenzimidoyl chloride (7a). ${ }^{1} \mathrm{H}$ NMR $\left(300 \mathrm{MHz}, \mathrm{CDCl}_{3}\right) \delta 8.09(\mathrm{~d}, J=7.0 \mathrm{~Hz}, 2 \mathrm{H})$, $7.50-7.29(\mathrm{~m}, 8 \mathrm{H}), 4.96(\mathrm{~s}, 2 \mathrm{H})$.

$\boldsymbol{N}$-(4-Nitrobenzyl)benzimidoyl chloride (7b). ${ }^{1} \mathrm{H}$ NMR $\left(300 \mathrm{MHz}, \mathrm{CDCl}_{3}\right) \delta 8.23(\mathrm{~d}, J=8.7$ $\mathrm{Hz}, 2 \mathrm{H}), 8.08$ (d, $J=7.1 \mathrm{~Hz}, 2 \mathrm{H}), 7.62$ (d, $J=8.6 \mathrm{~Hz}, 2 \mathrm{H}), 7.54-7.45$ (m, 4H), 5.00 (s, 2H).

$\mathrm{N}$-(4-Methylbenzyl)benzimidoyl chloride (7c). ${ }^{1} \mathrm{H}$ NMR $\left(300 \mathrm{MHz}, \mathrm{CDCl}_{3}\right) \delta 8.09(\mathrm{~d}, J=7.0$ $\mathrm{Hz}, 2 \mathrm{H}), 7.46-7.30$ (m, 7H), 4.95 (s, 2H), 2.32 (s, 3H).

$\boldsymbol{N}$-(4-Phenylbenzyl)benzimidoyl chloride (7d). ${ }^{1} \mathrm{H}$ NMR $\left(300 \mathrm{MHz}, \mathrm{CDCl}_{3}\right) \delta 8.12$ (d, $J=7.4$ $\mathrm{Hz}, 2 \mathrm{H}), 7.61-7.31$ (m, 2H), 4.99 (s, 2H).

$\boldsymbol{N}$-(4-Fluorobenzyl)benzimidoyl chloride (7e). ${ }^{1} \mathrm{H}$ NMR $\left(300 \mathrm{MHz}, \mathrm{CDCl}_{3}\right) \delta 8.06(\mathrm{~d}, J=7.0$ $\mathrm{Hz}, 2 \mathrm{H}), 7.52-7.38(\mathrm{~m}, 5 \mathrm{H}), 7.05(\mathrm{t}, J=8.7 \mathrm{~Hz}, 2 \mathrm{H}), 4.90\left(\mathrm{~s}, 2 \mathrm{H}, \mathrm{CH}_{2}\right)$.

$N$-(4-Bromobenzyl)benzimidoyl chloride (7f). ${ }^{1} \mathrm{H}$ NMR $\left(300 \mathrm{MHz}, \mathrm{CDCl}_{3}\right) \delta 8.18(\mathrm{~d}, J=7.5$ $\mathrm{Hz}, 2 \mathrm{H}), 7.50-7.32$ (m, 7H), 4.98 (s, 2H).

$\boldsymbol{N}$-(4-Chlorobenzyl)benzimidoyl chloride (7g). ${ }^{1} \mathrm{H}$ NMR $\left(300 \mathrm{MHz}, \mathrm{CDCl}_{3}\right) \delta 8.15(\mathrm{~d}, J=7.5$ $\mathrm{Hz}, 2 \mathrm{H}), 7.56-7.30$ (m, 7H), 4.96 (s, 2H).

\section{References.}

${ }^{1}$ Murray, A., W; Reid, R., G. Synthesis 1985, 1, 35 - 38.

${ }^{2}$ Katritzky, A., R.; Cai, Ch.; Singh, S., K.; J. Org. Chem. 2006, 9, 3375 - 3380.

${ }^{3}$ Elmorsy, A., R.; Saad, S.; Badawy, D., S.; Nour, M., A.; Kandeel, E., M.; Z. Naturforsch. B. 1994, 49, 3, 417 421.

${ }^{4}$ Darbeau, R., W.; White, E., H.; Song, F.; Darbeau, N., R.; Chou, J.; J. Org. Chem. 1999, 64, 16,5966 - 5978.

${ }^{5}$ Salehi, P.; Khodaeai, M., M.; Zolfigol, M., A.; Keyvan, A.; Synth. Commun. 2001, 31, 13, 1947 - 1952.

${ }^{6}$ Dube, D.; Scholte, A., A.; Tetrahedron Lett. 1999, 40, 2295 - 2298. 


\section{An ORTEP representation of exo-5a structure}
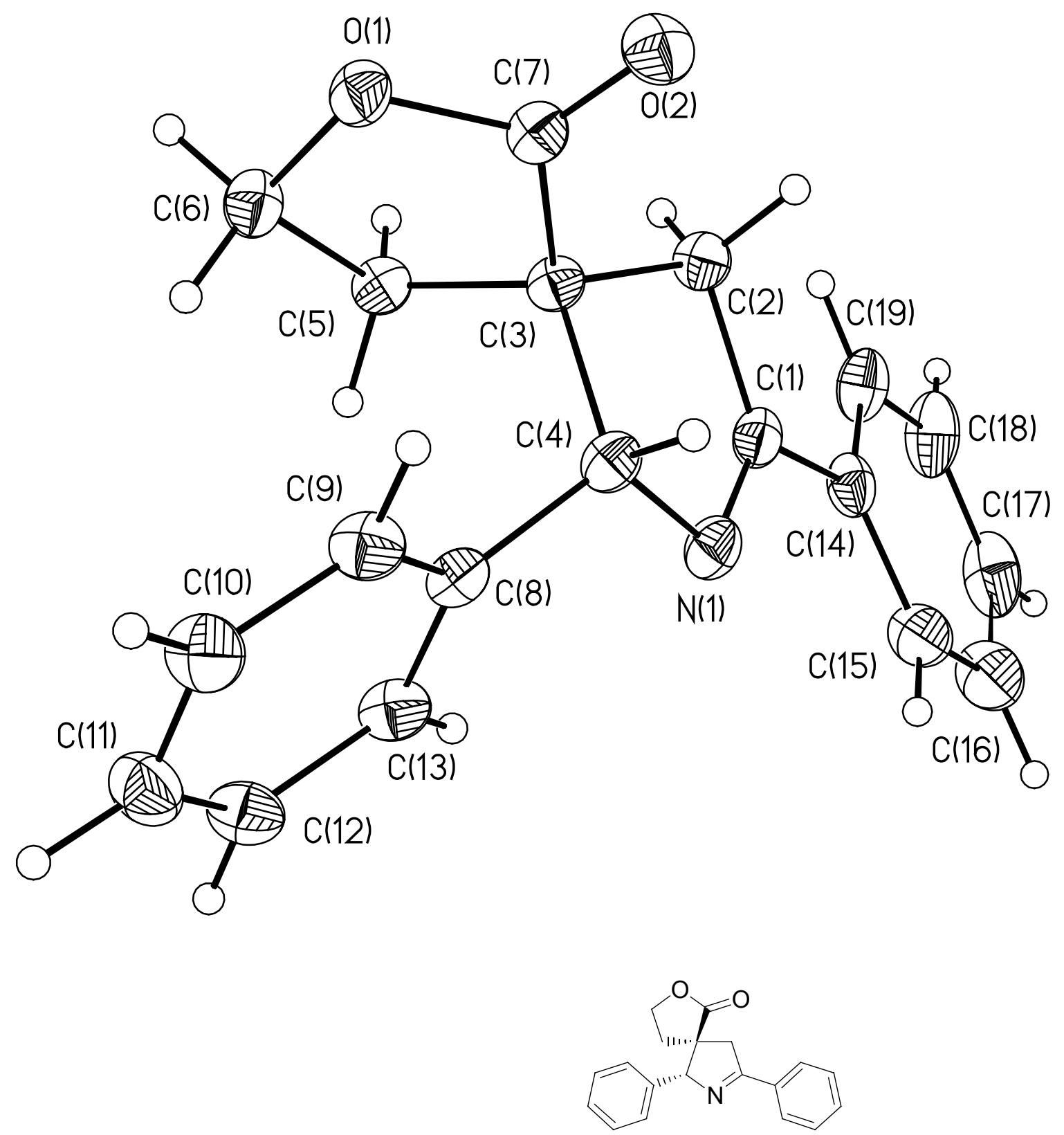

exo $-\mathbf{5 a}$ 


\section{${ }^{1} H$ NMR and ${ }^{13} \mathrm{C}$ NMR spectra}


6,8-Diphenyl-2-oxa-7-azaspiro[4.4]non-7-en-1-one (exo-5a) - ${ }^{1}$ H NMR

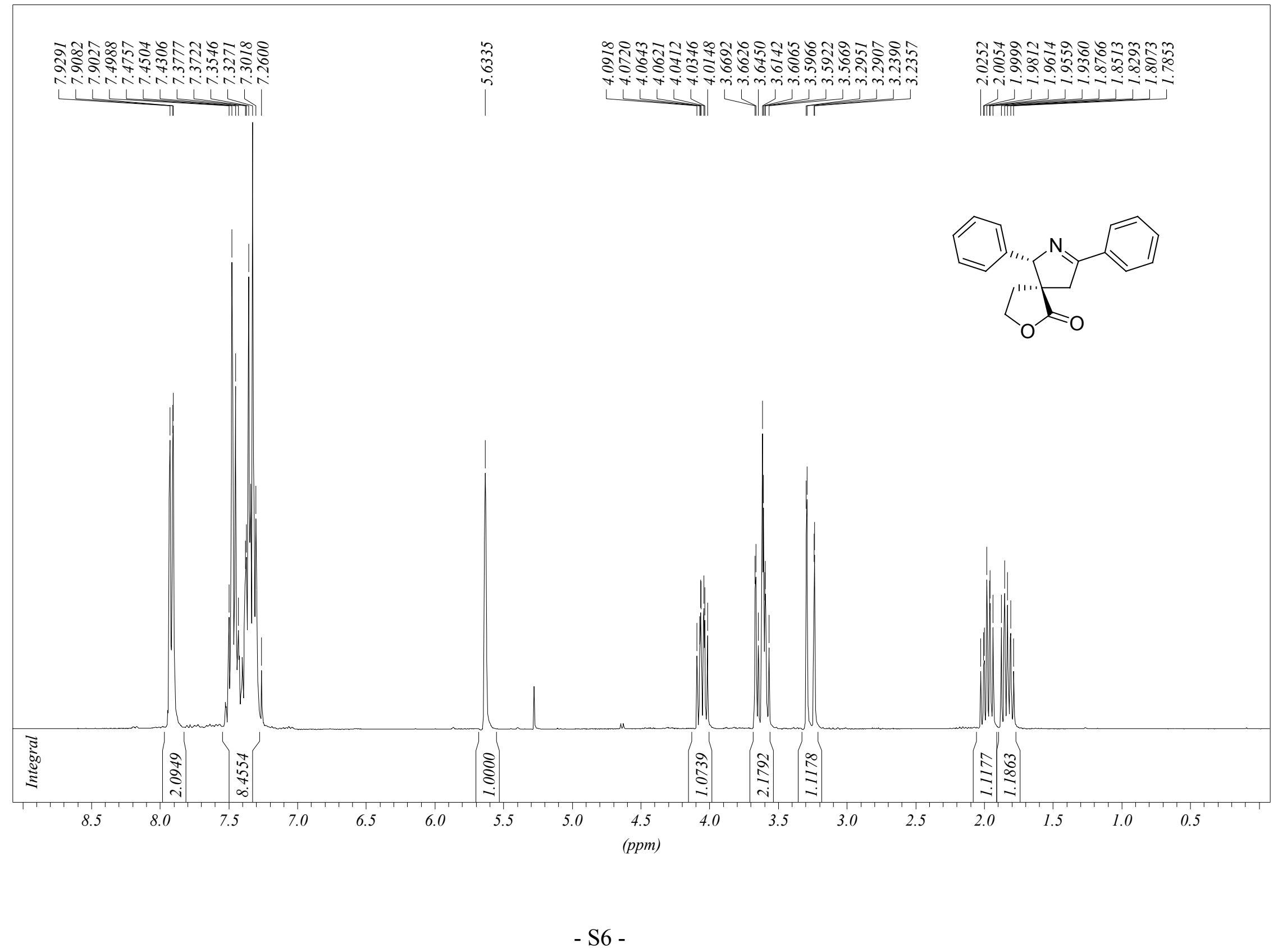


6,8-Diphenyl-2-oxa-7-azaspiro[4.4]non-7-en-1-one (exo-5a) - ${ }^{13} \mathrm{C}$ NMR




6-(4-Methylphenyl)-8-phenyl-2-oxa-7-azaspiro[4.4]non-7-en-1-one (exo-5c) - ${ }^{1} \mathrm{H}$ NMR

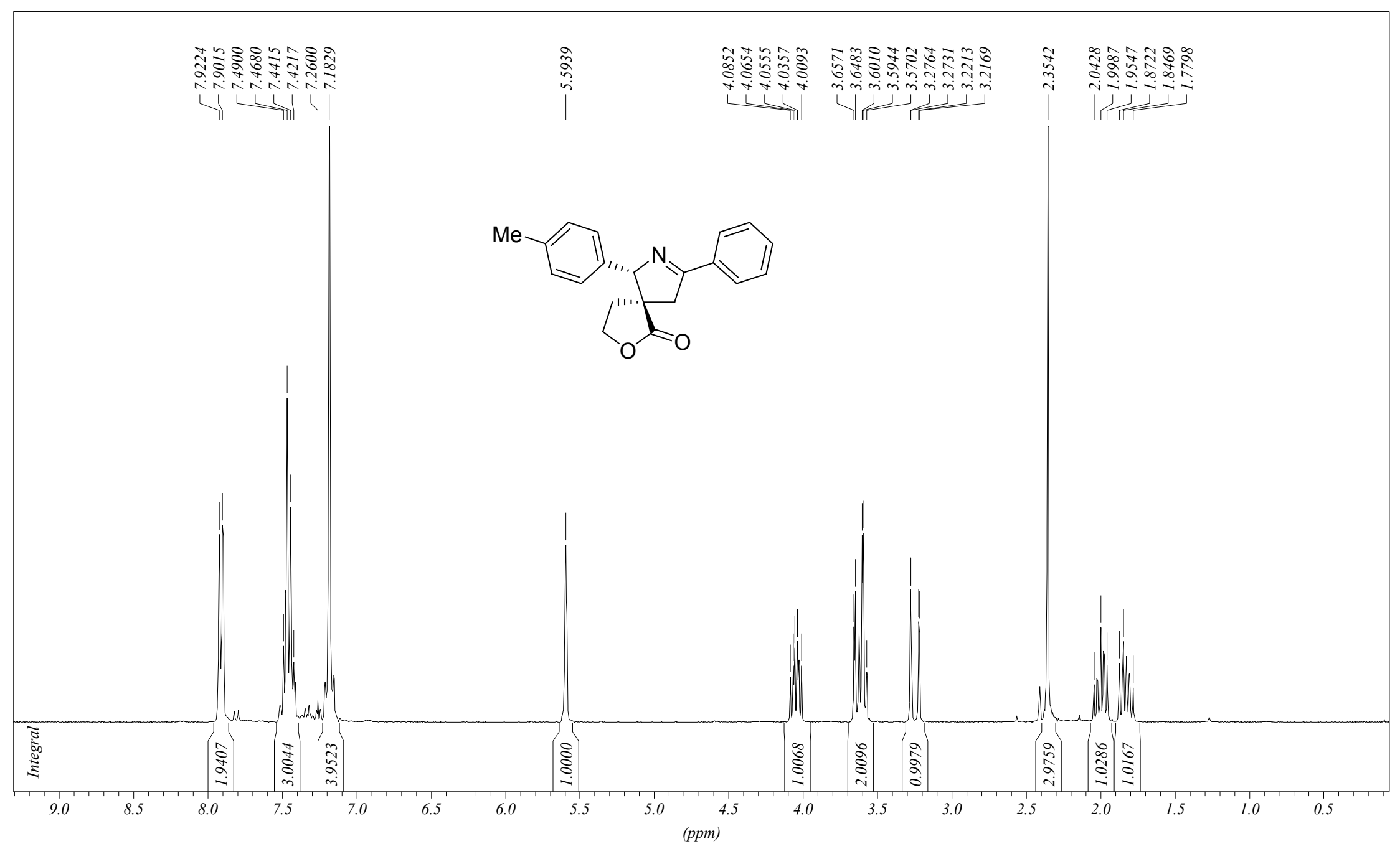


6-(4-Methylphenyl)-8-phenyl-2-oxa-7-azaspiro[4.4]non-7-en-1-one (exo-5c) - ${ }^{13} \mathrm{C}$ NMR




6-(4-Phenylphenyl)-8-phenyl-2-oxa-7-azaspiro[4.4]non-7-en-1-one (exo-5d) - ${ }^{1} \mathrm{H}$ NMR

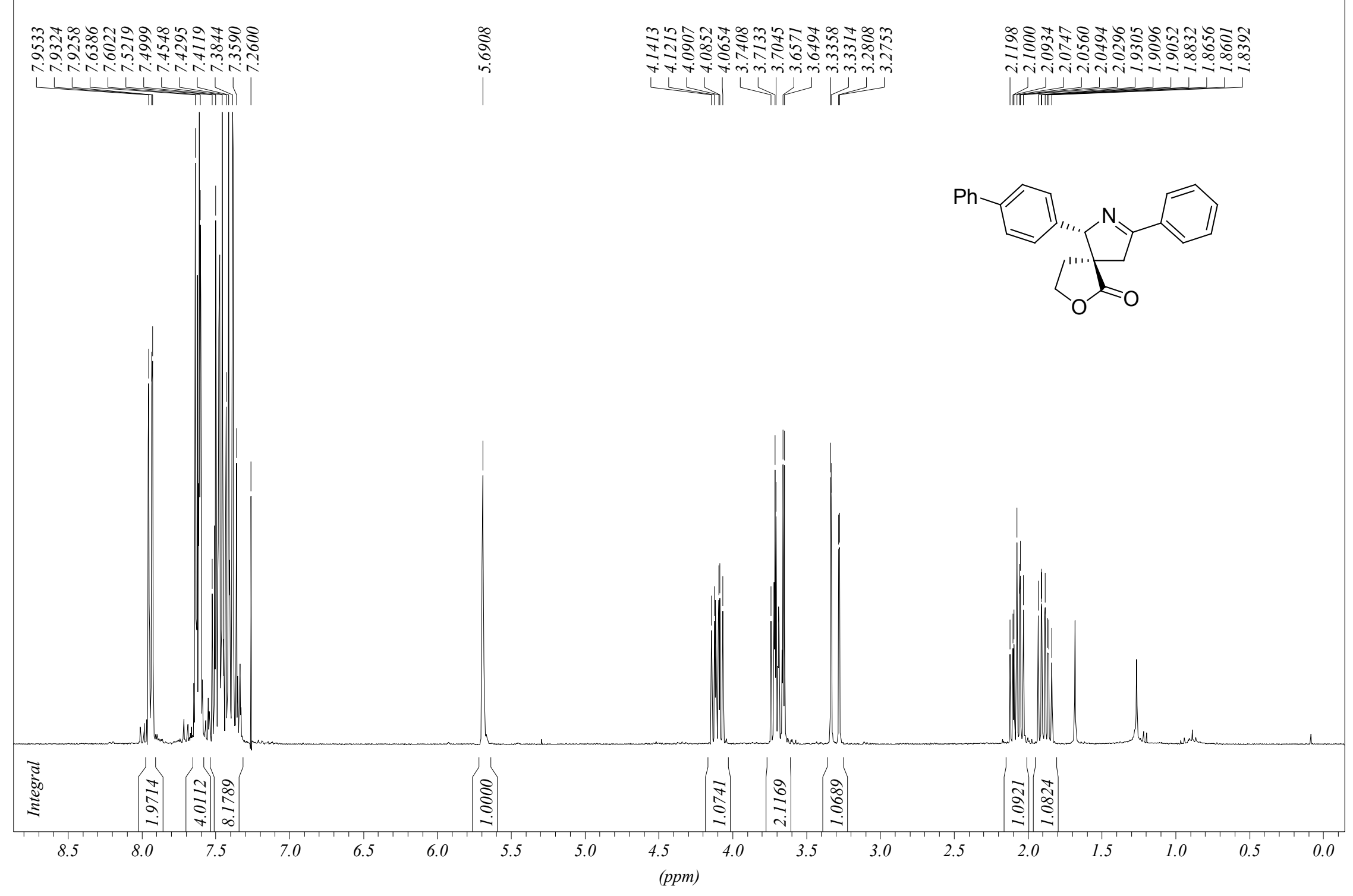


6-(4-Phenylphenyl)-8-phenyl-2-oxa-7-azaspiro[4.4]non-7-en-1-one (exo-5d) - ${ }^{13} \mathrm{C}$ NMR

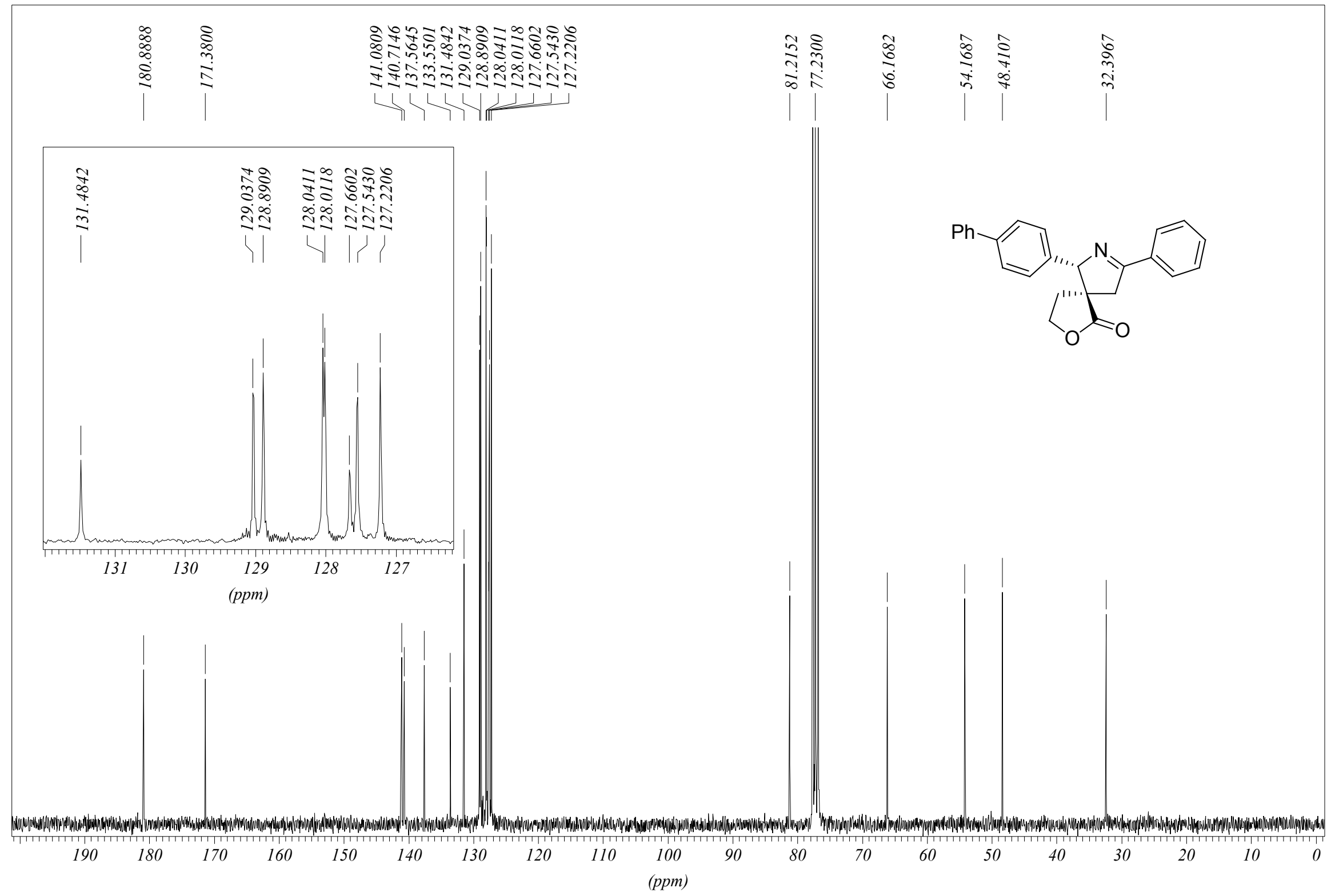




\section{6-(4-Fluorophenyl)-8-phenyl-2-oxa-7-azaspiro[4.4] non-7-en-1-one (exo-5e) - ${ }^{1} \mathrm{H}$ NMR}

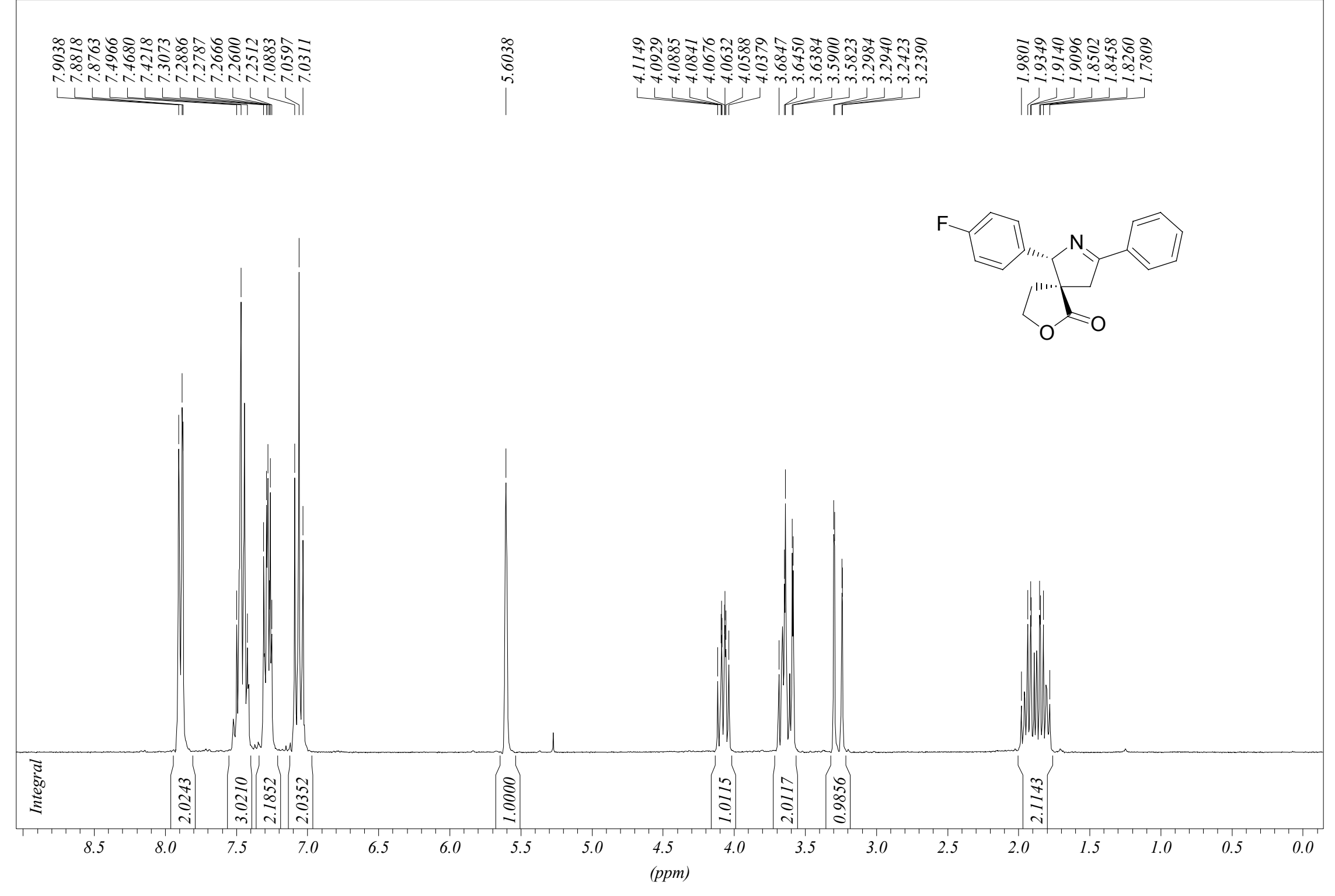


6-(4-Fluorophenyl)-8-phenyl-2-oxa-7-azaspiro[4.4]non-7-en-1-one (exo-5e) - ${ }^{13} \mathrm{C}$ NMR

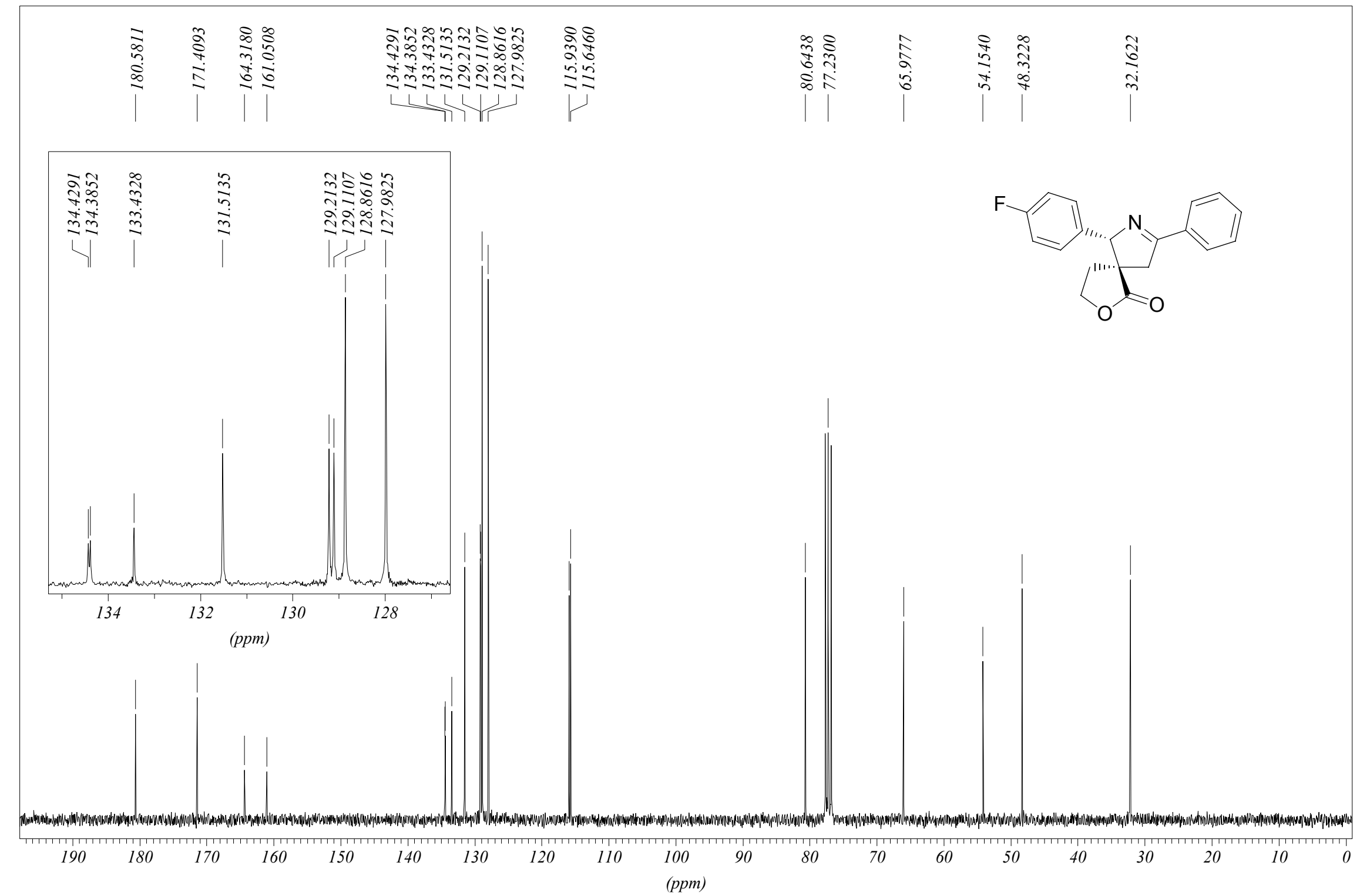


6-(4-Bromophenyl)-8-phenyl-2-oxa-7-azaspiro[4.4] non-7-en-1-one (exo-5f) - ${ }^{1} \mathrm{H}$ NMR

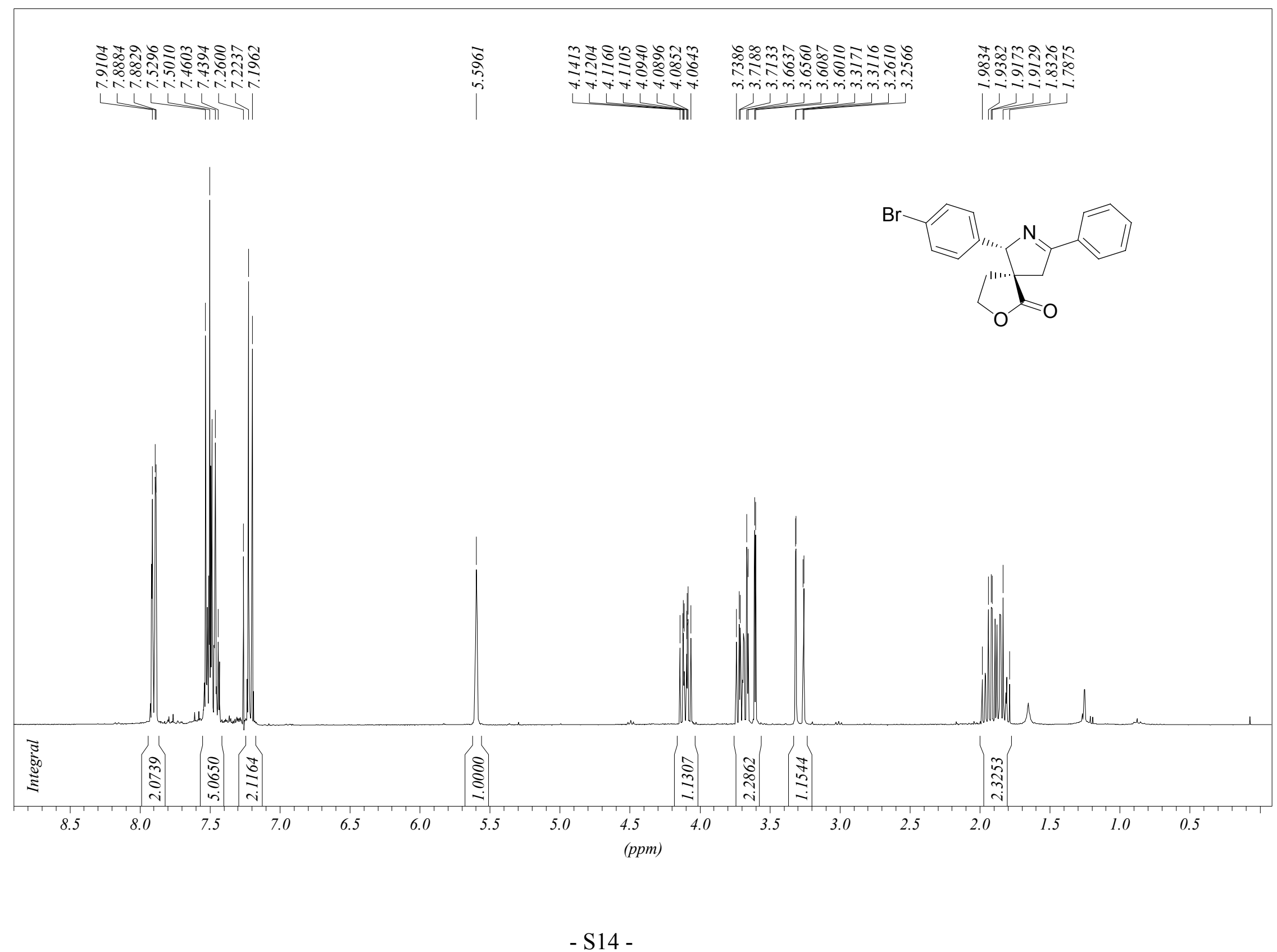


6-(4-Bromophenyl)-8-phenyl-2-oxa-7-azaspiro[4.4]non-7-en-1-one (exo-5f) - ${ }^{13} \mathrm{C}$ NMR




6-(4-Chlorophenyl)-8-phenyl-2-oxa-7-azaspiro[4.4]non-7-en-1-one (exo-5g) - ${ }^{1} \mathrm{H}$ NMR

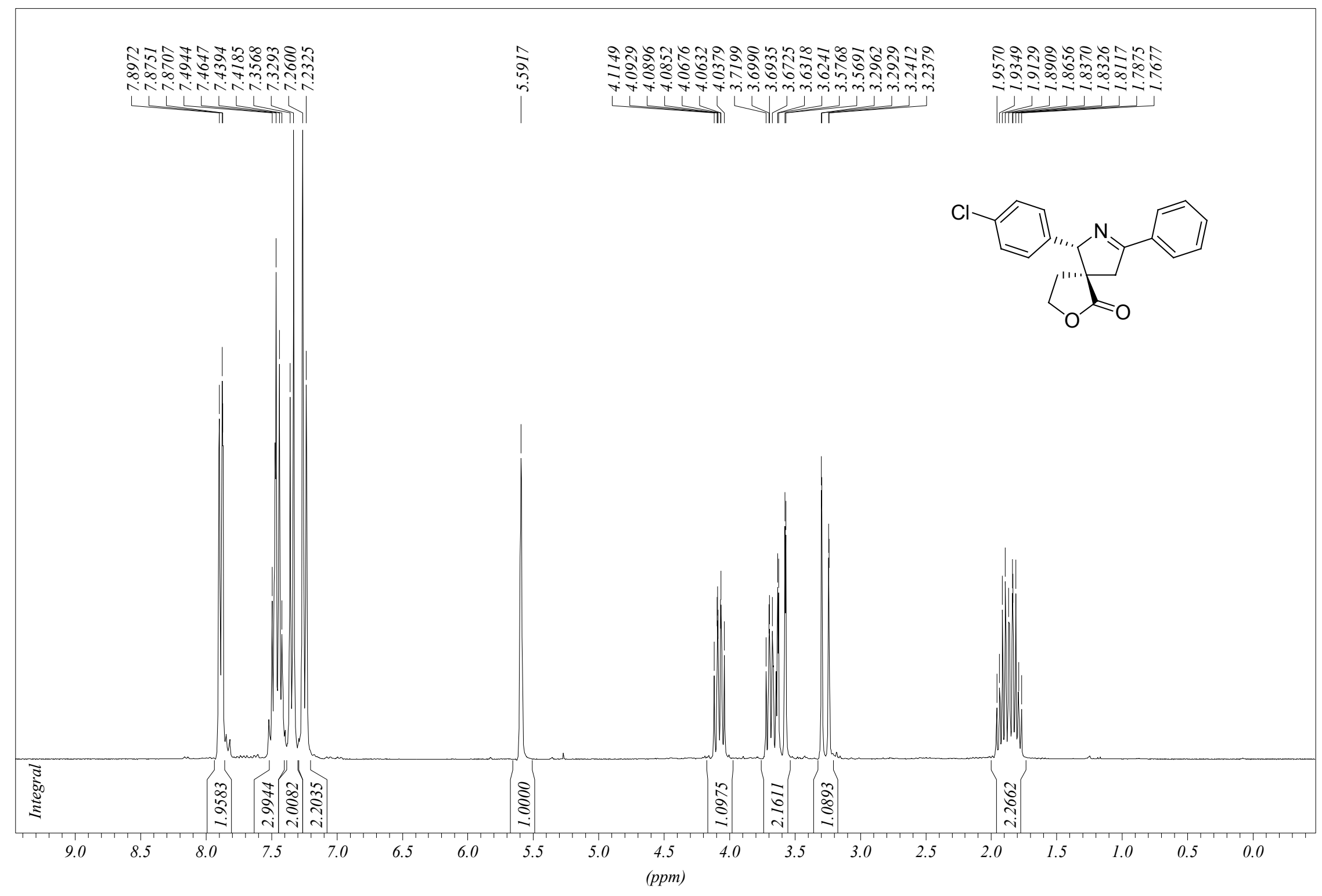


6-(4-Chlorophenyl)-8-phenyl-2-oxa-7-azaspiro[4.4]non-7-en-1-one (exo-5g) - ${ }^{13} \mathrm{C}$ NMR

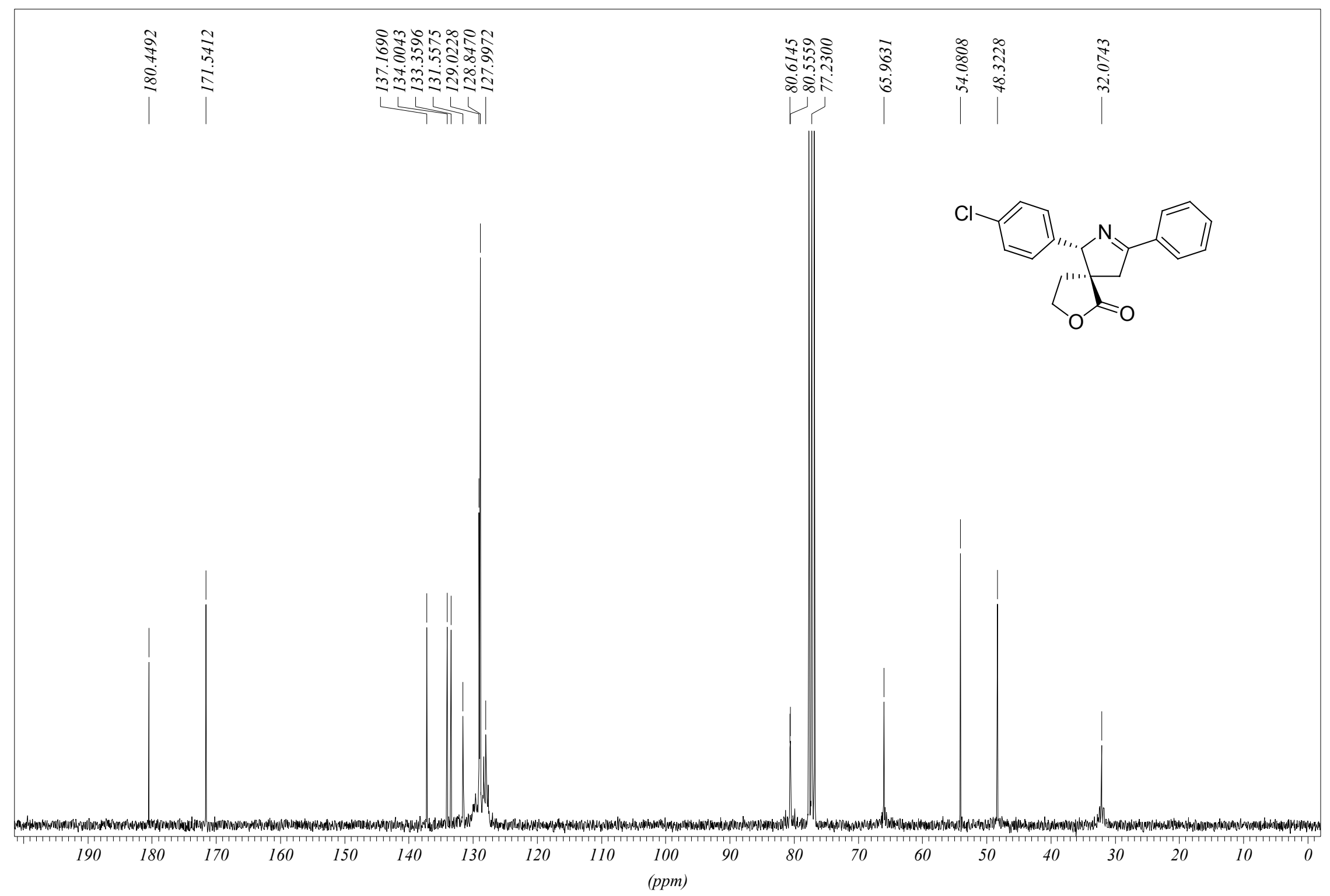


${ }^{1} \mathrm{H}$ NMR Spectra of crude reaction mixtures for exo/endo ratios determination 
6,8-Diphenyl-2-oxa-7-azaspiro[4.4]non-7-en-1-one (exo-5a) - ${ }^{1} \mathrm{H}$ NMR spectrum of the crude reaction mixture




6-(4-Nitrophenyl)-8-phenyl-2-oxa-7-azaspiro[4.4]non-7-en-1-one (exo-5b) - ${ }^{1} \mathrm{H}$ NMR spectrum of the crude reaction mixture.

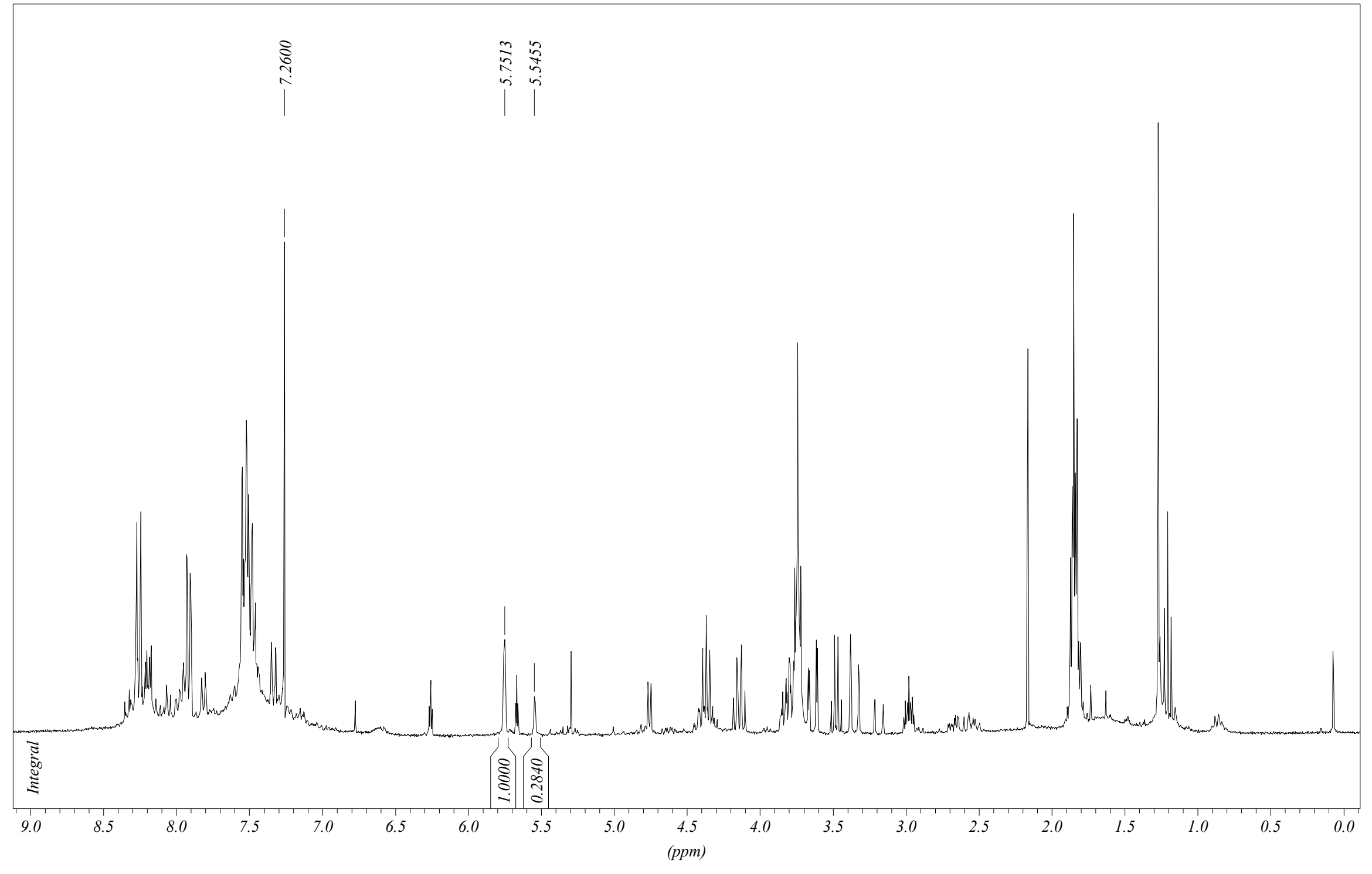


6-(4-Methylphenyl)-8-phenyl-2-oxa-7-azaspiro[4.4]non-7-en-1-one (exo-5c) - ${ }^{1} \mathrm{H}$ NMR spectrum of the crude reaction mixture

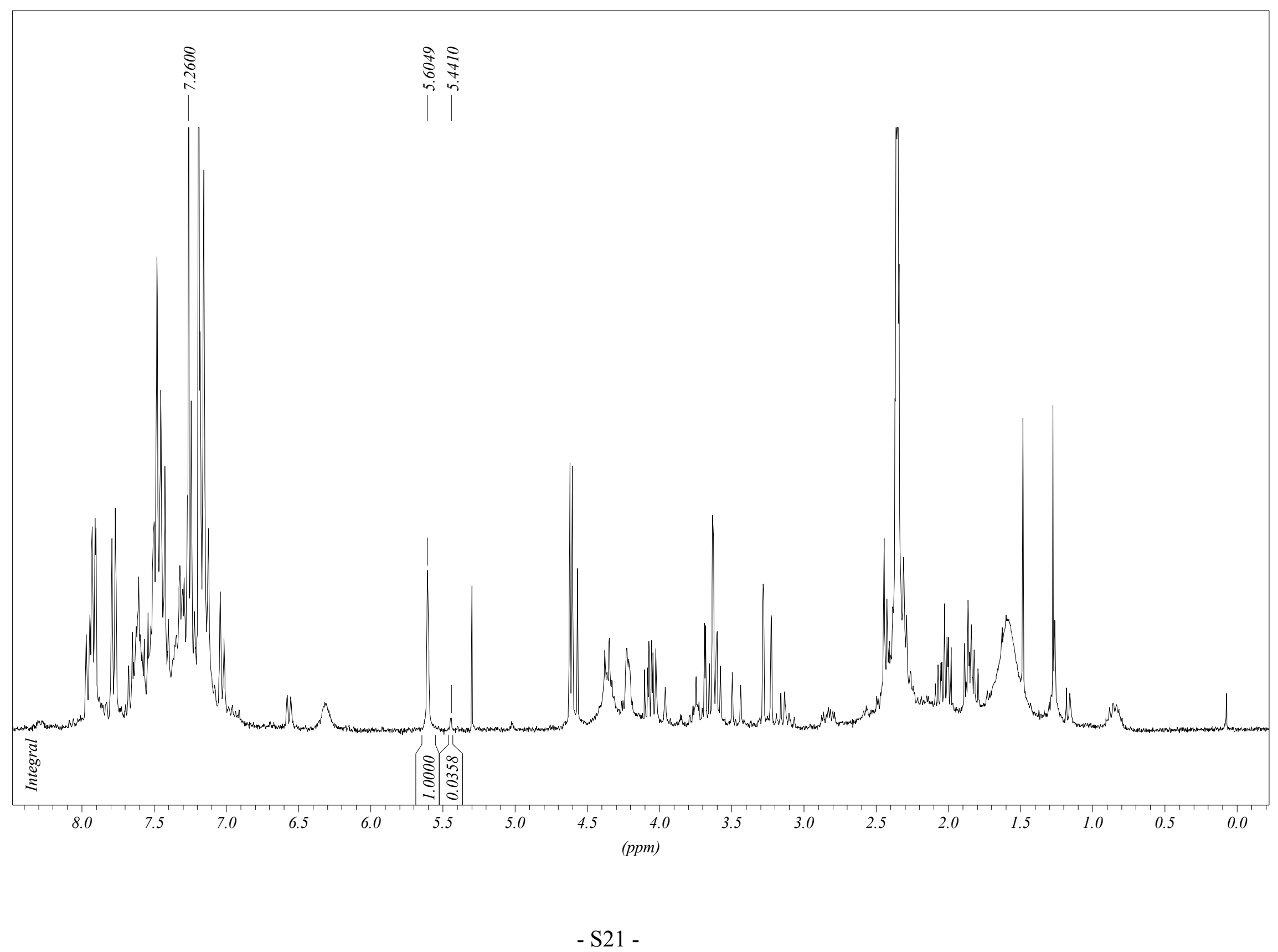


6-(4-Phenylphenyl)-8-phenyl-2-oxa-7-azaspiro[4.4]non-7-en-1-one (exo-5d) - ${ }^{1} \mathrm{H}$ NMR spectrum of the crude reaction mixture

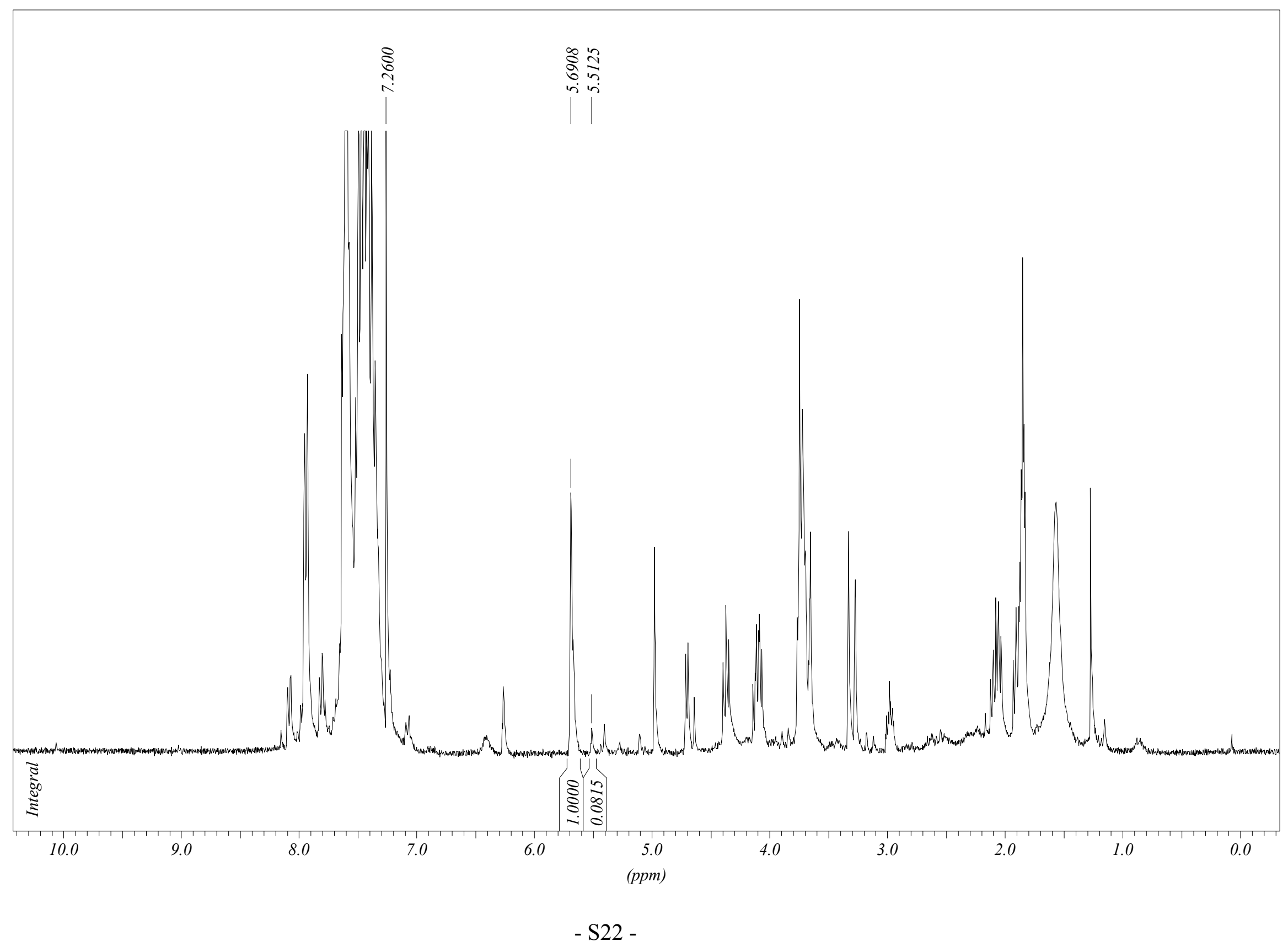


6-(4-Fluorophenyl)-8-phenyl-2-oxa-7-azaspiro[4.4]non-7-en-1-one (exo-5e) - ${ }^{1} \mathrm{H}$ NMR spectrum of the crude reaction mixture

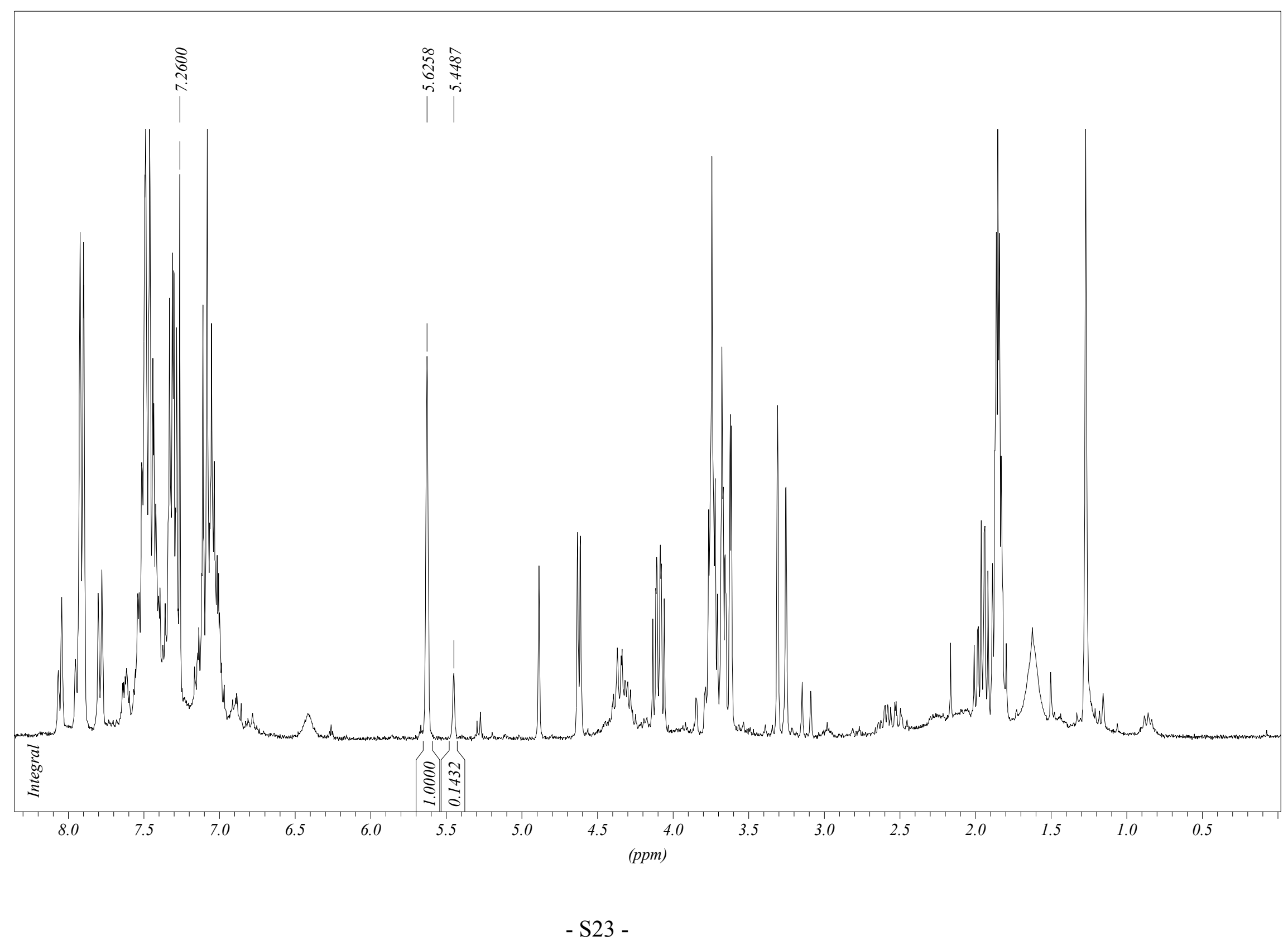


6-(4-Bromophenyl)-8-phenyl-2-oxa-7-azaspiro[4.4]non-7-en-1-one (exo-5f) - ${ }^{1} \mathrm{H}$ NMR spectrum of the crude reaction mixture

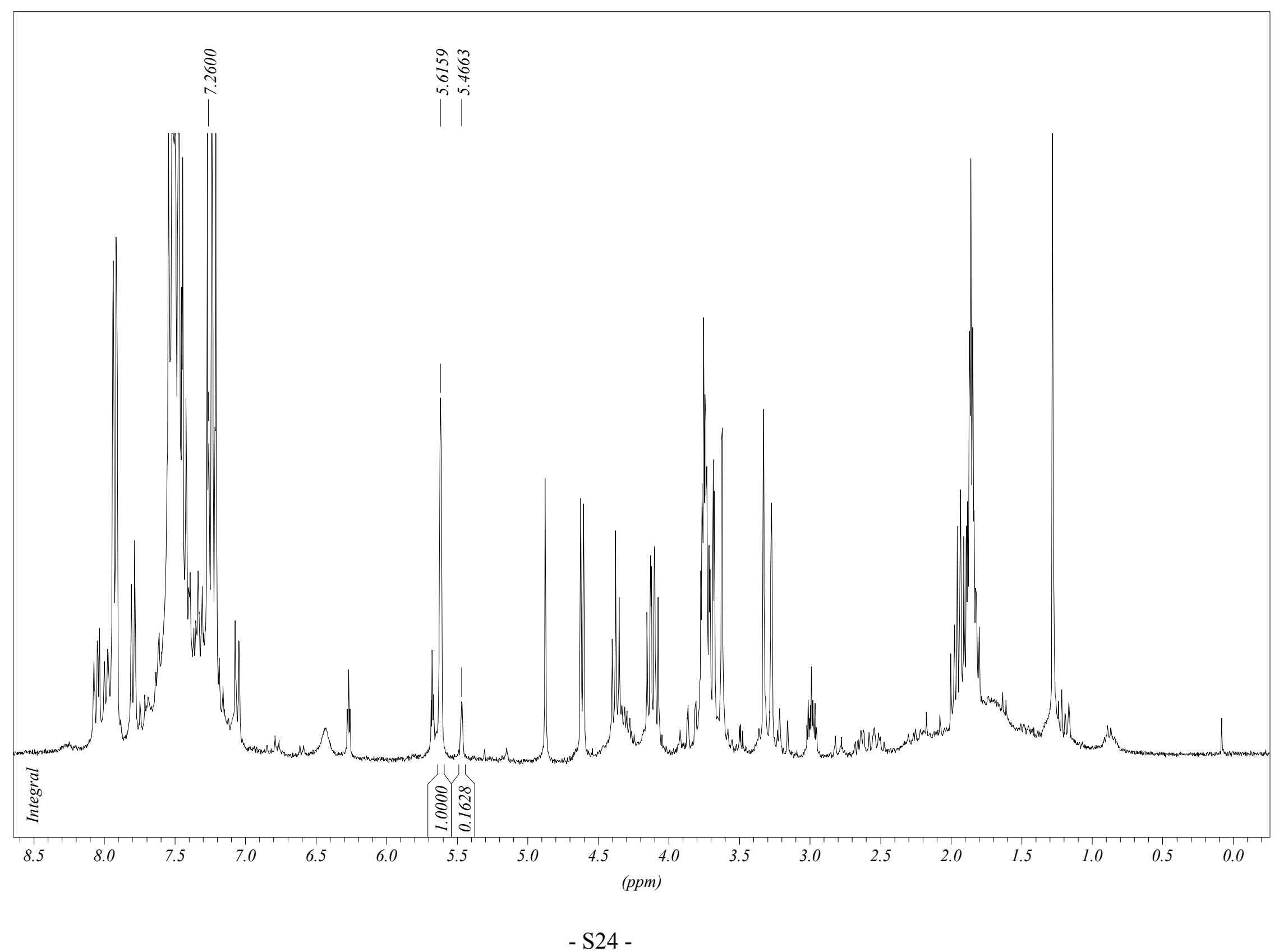


6-(4-Chlorophenyl)-8-phenyl-2-oxa-7-azaspiro[4.4]non-7-en-1-one (exo-5g) - ${ }^{1} \mathrm{H}$ NMR spectrum of the crude reaction mixture




Hammett-like plots correlating $\log ($ d.e. $)$ with substituent sigma values $\sigma_{p}^{\circ}(\bullet)$ and $\sigma_{m} .(\bullet)$.

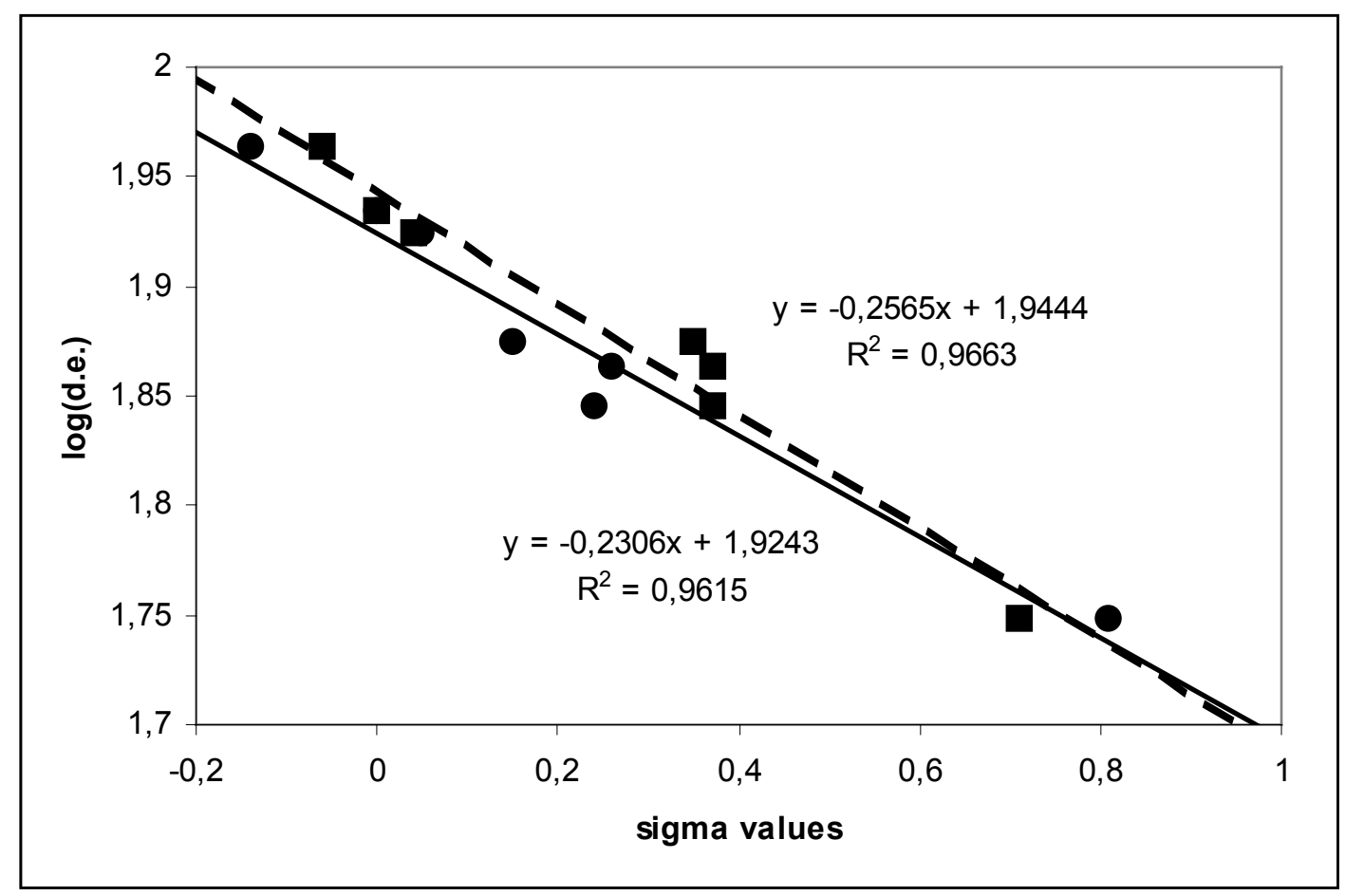

\begin{tabular}{cccccc}
\hline product & substitent & d.e (\%) & $\log ($ d.e. $)$ & $\sigma_{\boldsymbol{p}}^{\circ}$ & $\sigma_{\boldsymbol{m}}$ \\
\hline $\mathbf{5 a}$ & $\mathrm{H}$ & 86 & 1,9344 & 0 & 0 \\
$\mathbf{5 b}$ & $\mathrm{NO}_{2}$ & 56 & 1,7481 & 0,81 & 0,71 \\
$\mathbf{5 c}$ & $\mathrm{CH}_{3}$ & 92 & 1,9637 & $-0,14$ & $-0,06$ \\
$\mathbf{5 d}$ & $\mathrm{Ph}$ & 85 & 1,9294 & 0,05 & 0,04 \\
$\mathbf{5 e}$ & $\mathrm{F}$ & 75 & 1,8750 & 0,15 & 0,35 \\
$\mathbf{5 f}$ & $\mathrm{Br}$ & 73 & 1,8633 & 0,26 & 0,37 \\
$\mathbf{5 g}$ & $\mathrm{Cl}$ & 70 & 1,8450 & 0,24 & 0,37 \\
\hline
\end{tabular}




\section{Cartesian coordinates of transition states optimized by MPW1K/aug-cc-pVDZ.}

\begin{tabular}{|c|c|c|c|}
\hline \multicolumn{4}{|c|}{ Product 5a-exo-TS } \\
\hline $\mathrm{H}$ & 1.77810200 & -1.96245800 & 2.52321800 \\
\hline $\mathrm{C}$ & -0.53484500 & -2.12355900 & 0.81269700 \\
\hline $\mathrm{H}$ & -0.95505700 & -1.99178100 & 1.80208700 \\
\hline $\mathrm{C}$ & 0.79423200 & -1.97818300 & 0.59696700 \\
\hline $\mathrm{H}$ & 1.82005700 & -0.41476100 & 1.68026500 \\
\hline $\mathrm{C}$ & 3.13604800 & -1.92069000 & 0.81543900 \\
\hline $\mathrm{H}$ & 3.60639500 & -2.78566000 & 1.28908500 \\
\hline $\mathrm{H}$ & 3.86873900 & -1.11772100 & 0.74348200 \\
\hline $\mathrm{O}$ & 2.77568500 & -2.29629100 & -0.51133400 \\
\hline $\mathrm{C}$ & 1.85268200 & -1.49917300 & 1.53733200 \\
\hline $\mathrm{C}$ & 0.61799100 & 0.37620400 & -0.99733600 \\
\hline $\mathrm{H}$ & 0.83161200 & -0.19768200 & -1.89320900 \\
\hline $\mathrm{C}$ & -1.52450600 & -0.18836700 & 0.02421400 \\
\hline $\mathrm{N}$ & -0.58956300 & 0.32625500 & -0.55449200 \\
\hline $\mathrm{C}$ & -2.93866000 & 0.14968500 & 0.00386200 \\
\hline $\mathrm{H}$ & -5.14683200 & 2.37535900 & -1.29351800 \\
\hline $\mathrm{C}$ & -5.65385300 & 0.74451700 & 0.01145800 \\
\hline $\mathrm{C}$ & -3.82726000 & -0.63142500 & 0.73938500 \\
\hline $\mathrm{C}$ & -3.42324500 & 1.23592500 & -0.73152100 \\
\hline $\mathrm{C}$ & -4.77393100 & 1.53163900 & -0.72370700 \\
\hline $\mathrm{C}$ & -5.18166500 & -0.33676400 & 0.74099700 \\
\hline $\mathrm{H}$ & -3.44697600 & -1.47301500 & 1.30570600 \\
\hline $\mathrm{H}$ & -2.72975200 & 1.84072400 & -1.30429600 \\
\hline $\mathrm{C}$ & 2.33968200 & 2.96229200 & 1.10965300 \\
\hline $\mathrm{C}$ & 1.60938100 & 1.29574100 & -0.47800200 \\
\hline $\mathrm{C}$ & 3.59859200 & 2.98479700 & 0.52174400 \\
\hline $\mathrm{C}$ & 1.35202900 & 2.12693800 & 0.61723900 \\
\hline $\mathrm{C}$ & 2.87869900 & 1.32503000 & -1.06347200 \\
\hline $\mathrm{C}$ & 1.43365100 & -2.41375700 & -0.64189800 \\
\hline $\mathrm{O}$ & 0.93742000 & -2.82292400 & -1.65827200 \\
\hline $\mathrm{H}$ & -6.71312300 & 0.97724300 & 0.01339700 \\
\hline $\mathrm{H}$ & 0.36858500 & 2.12094900 & 1.07362900 \\
\hline $\mathrm{H}$ & 3.08974500 & 0.67899600 & -1.90877700 \\
\hline $\mathrm{H}$ & 4.84248000 & 2.17435000 & -1.03064000 \\
\hline $\mathrm{H}$ & 2.12621900 & 3.60485300 & 1.95686100 \\
\hline $\mathrm{C}$ & 3.86284400 & 2.16204100 & -0.56575100 \\
\hline $\mathrm{H}$ & -5.86902400 & -0.95012300 & 1.31201800 \\
\hline $\mathrm{H}$ & 4.36873300 & 3.64297100 & 0.90779600 \\
\hline $\mathrm{H}$ & -1.12500900 & -2.66966300 & 0.08539600 \\
\hline
\end{tabular}




\section{Product 5a - endo-TS}

$\begin{array}{lrrr}\mathrm{H} & -0.67742400 & 2.68316700 & -1.96429300 \\ \mathrm{C} & 0.56553400 & 2.20033700 & 0.67844600 \\ \mathrm{H} & 0.70508800 & 1.84555700 & 1.69227000 \\ \mathrm{C} & -0.68956400 & 2.23528200 & 0.17274600 \\ \mathrm{H} & -1.16696500 & 4.01918200 & -0.92664100 \\ \mathrm{C} & -2.66188400 & 2.44763300 & -1.07953400 \\ \mathrm{H} & -2.80304600 & 1.64093500 & -1.80459700 \\ \mathrm{H} & -3.37786300 & 3.23926300 & -1.29910000 \\ \mathrm{O} & -2.96359700 & 1.93716700 & 0.21109700 \\ \mathrm{C} & -1.21187800 & 2.93159600 & -1.04491600 \\ \mathrm{C} & -0.61504700 & -0.40511200 & -1.09696500 \\ \mathrm{H} & -0.82291600 & 0.00706600 & -2.08028900 \\ \mathrm{C} & 1.53023500 & 0.26341300 & -0.12276700 \\ \mathrm{~N} & 0.59069800 & -0.27733700 & -0.66763700 \\ \mathrm{C} & 2.93328100 & -0.11893400 & -0.08771900 \\ \mathrm{H} & 5.12325600 & -2.41017900 & -1.29921900 \\ \mathrm{C} & 5.62799000 & -0.79156200 & 0.02181100 \\ \mathrm{C} & 3.81628500 & 0.63915900 & 0.67818600 \\ \mathrm{C} & 3.41282600 & -1.22211400 & -0.80075700 \\ \mathrm{C} & 4.75396900 & -1.55407600 & -0.74572400 \\ \mathrm{C} & 5.15990800 & 0.30287700 & 0.73416500 \\ \mathrm{H} & 3.43854300 & 1.49040800 & 1.23179800 \\ \mathrm{H} & 2.72268700 & -1.81263900 & -1.39235000 \\ \mathrm{C} & -2.38103300 & -2.65732400 & 1.32878300 \\ \mathrm{C} & -1.59782600 & -1.28236500 & -0.48777700 \\ \mathrm{C} & -3.54872400 & -2.93388100 & 0.62883100 \\ \mathrm{C} & -1.41162300 & -1.83847200 & 0.77961000 \\ \mathrm{C} & -2.77699300 & -1.56003100 & -1.18258000 \\ \mathrm{C} & -1.82748900 & 1.69908400 & 0.91329800 \\ \mathrm{O} & -1.85191100 & 1.15479900 & 1.98283700 \\ \mathrm{H} & 6.67915600 & -1.05517300 & 0.06417800 \\ \mathrm{H} & -0.51194800 & -1.61270400 & 1.33876100 \\ \mathrm{H} & -2.93202000 & -1.13084400 & -2.16739800 \\ \mathrm{H} & -4.65580300 & -2.58613000 & -1.17890700 \\ \mathrm{H} & -2.23069000 & -3.07543700 & 2.31762400 \\ \mathrm{C} & -3.74466600 & -2.37952200 & -0.62861900 \\ \mathrm{H} & 5.84195600 & 0.89434600 & 1.33393600 \\ \mathrm{H} & -4.30687100 & -3.57377500 & 1.06604100 \\ \mathrm{H} & 1.36423100 & 2.78664900 & 0.23906500\end{array}$




\section{Product 5b-exo-TS}

$\begin{array}{lrrr}\mathrm{H} & -0.44733400 & 2.39574700 & 2.63097900 \\ \mathrm{C} & 1.87873100 & 1.95965600 & 0.99563400 \\ \mathrm{H} & 2.16935200 & 1.58813000 & 1.97082300 \\ \mathrm{C} & 0.58992000 & 2.28783800 & 0.73778800 \\ \mathrm{H} & -0.96049000 & 1.05734300 & 1.60764800 \\ \mathrm{C} & -1.64390200 & 3.00804600 & 0.91136900 \\ \mathrm{H} & -1.82135800 & 3.92299500 & 1.48067300 \\ \mathrm{H} & -2.59910400 & 2.51761100 & 0.72892400 \\ \mathrm{O} & -1.10332800 & 3.38312200 & -0.35374000 \\ \mathrm{C} & -0.61936900 & 2.09709600 & 1.59525200 \\ \mathrm{C} & 0.10461800 & 0.23806500 & -1.14402200 \\ \mathrm{H} & 0.15610900 & 0.94485800 & -1.96472500 \\ \mathrm{C} & 2.24053300 & -0.06741500 & -0.01535900 \\ \mathrm{~N} & 1.23251500 & -0.18324400 & -0.67018000 \\ \mathrm{C} & 3.49264600 & -0.79680800 & 0.00731800 \\ \mathrm{H} & 5.11718700 & -3.34370000 & -1.53510500 \\ \mathrm{C} & 5.90713500 & -2.16544600 & 0.07980700 \\ \mathrm{C} & 4.47347900 & -0.43887500 & 0.92988900 \\ \mathrm{C} & 3.73079300 & -1.84839800 & -0.88376500 \\ \mathrm{C} & 4.93348100 & -2.52867700 & -0.84434100 \\ \mathrm{C} & 5.67829100 & -1.12234000 & 0.96541000 \\ \mathrm{H} & 4.28646300 & 0.37878300 & 1.61492700 \\ \mathrm{H} & 2.96554000 & -2.12148900 & -1.60082300 \\ \mathrm{C} & -2.48598600 & -1.85399300 & 0.57392300 \\ \mathrm{C} & -1.15867400 & -0.34679700 & -0.77108900 \\ \mathrm{C} & -3.62711100 & -1.35911500 & -0.03967600 \\ \mathrm{C} & -1.25681400 & -1.34650300 & 0.20723400 \\ \mathrm{C} & -2.33143200 & 0.13084100 & -1.37262000 \\ \mathrm{C} & 0.20498100 & 3.05749300 & -0.44617800 \\ \mathrm{O} & 0.87143600 & 3.38908800 & -1.38979700 \\ \mathrm{H} & 6.85016100 & -2.70020100 & 0.10732900 \\ \mathrm{H} & -0.35726200 & -1.73241900 & 0.67139200 \\ \mathrm{H} & -2.26611800 & 0.90877900 & -2.12439700 \\ \mathrm{H} & -4.47841000 & -0.01230600 & -1.46510900 \\ \mathrm{H} & -2.58020700 & -2.62816100 & 1.32344600 \\ \mathrm{H} & -3.56461900 & -0.37012900 & -1.01037100 \\ \mathrm{H} & 6.43968100 & -0.84065600 & 1.68324100 \\ \mathrm{H} & -5.95698600 & 2.36474200 & 0.35812200 \\ \mathrm{H} & & -1.89159300 & 0.34380700 \\ \mathrm{H} & -2.75388900 & 1.19037600 \\ \mathrm{H} & -1.44124800 & -0.20433500\end{array}$




\section{Product 5b-endo-TS}

$\begin{array}{lrrr}\mathrm{H} & 1.13297200 & 3.32744900 & -1.57162400 \\ \mathrm{C} & 1.88859700 & 1.99932800 & 0.96830700 \\ \mathrm{H} & 1.79842600 & 1.44639500 & 1.89587600 \\ \mathrm{C} & 0.76908400 & 2.53548600 & 0.42877700 \\ \mathrm{H} & 1.01831600 & 4.53854600 & -0.29918300 \\ \mathrm{C} & -0.89350800 & 3.63785400 & -0.81062500 \\ \mathrm{H} & -1.22558500 & 3.09382900 & -1.69921900 \\ \mathrm{H} & -1.28020600 & 4.65493800 & -0.86538100 \\ \mathrm{O} & -1.47218600 & 3.01587600 & 0.32826200 \\ \mathrm{C} & 0.62481000 & 3.57492500 & -0.63752700 \\ \mathrm{C} & 0.11030000 & 0.28371900 & -1.27952800 \\ \mathrm{H} & 0.12129400 & 0.88238100 & -2.18460200 \\ \mathrm{C} & 2.26582400 & 0.02885700 & -0.15369600 \\ \mathrm{~N} & 1.25992300 & -0.07611700 & -0.81136100 \\ \mathrm{C} & 3.48633300 & -0.74996900 & -0.08495600 \\ \mathrm{H} & 5.09344200 & -3.32575600 & -1.59726100 \\ \mathrm{C} & 5.84219300 & -2.20965600 & 0.08015700 \\ \mathrm{C} & 4.43156300 & -0.44623600 & 0.89284200 \\ \mathrm{C} & 3.72965100 & -1.79416700 & -0.98311000 \\ \mathrm{C} & 4.90496000 & -2.51713800 & -0.90024800 \\ \mathrm{C} & 5.60534400 & -1.17793200 & 0.97696600 \\ \mathrm{H} & 4.23666100 & 0.36150800 & 1.58763700 \\ \mathrm{H} & 2.98953000 & -2.02907100 & -1.73915600 \\ \mathrm{C} & -2.41631200 & -1.65175500 & 0.69703600 \\ \mathrm{C} & -1.13627800 & -0.30108100 & -0.83711600 \\ \mathrm{C} & -3.55814700 & -1.35161100 & -0.02952000 \\ \mathrm{C} & -1.20871800 & -1.12466600 & 0.29154300 \\ \mathrm{C} & -2.30879200 & -0.01050300 & -1.54388200 \\ \mathrm{C} & -0.55166700 & 2.28808600 & 1.00509800 \\ \mathrm{O} & -0.85395100 & 1.59667900 & 1.93774400 \\ \mathrm{H} & 6.76195700 & -2.78056000 & 0.14438800 \\ \mathrm{H} & -0.31285000 & -1.34133800 & 0.85867200 \\ \mathrm{H} & -2.26382600 & 0.62866100 & -2.41851400 \\ \mathrm{H} & -4.43715200 & -0.31782800 & -1.68062700 \\ \mathrm{H} & -2.49305300 & -2.28532500 & 1.56982200 \\ \mathrm{H} & -3.52213900 & -0.53056800 & -1.14517900 \\ \mathrm{H} & 6.33620600 & -0.94263400 & 1.74173400 \\ \mathrm{H} & -8.87253300 & 2.35826300 & 0.68890100 \\ \mathrm{H} & & -1.91407700 & 0.38880200 \\ \mathrm{H} & -2860800 & -1.62349300 & -0.25327300\end{array}$




\section{Product 5c-exo-TS}

$\begin{array}{lrrr}\mathrm{H} & -1.28113600 & 2.27434300 & 2.55094800 \\ \mathrm{C} & 1.10174000 & 2.13902300 & 0.92741900 \\ \mathrm{H} & 1.45999700 & 1.89284900 & 1.91919800 \\ \mathrm{C} & -0.22438400 & 2.21639700 & 0.66533300 \\ \mathrm{H} & -1.50629700 & 0.77865900 & 1.64586700 \\ \mathrm{C} & -2.55972500 & 2.48637300 & 0.79505700 \\ \mathrm{H} & -2.94058900 & 3.37730800 & 1.29955400 \\ \mathrm{H} & -3.38296600 & 1.78924400 & 0.64236700 \\ \mathrm{O} & -2.08907300 & 2.88460100 & -0.48981000 \\ \mathrm{C} & -1.37921400 & 1.86088200 & 1.54502900 \\ \mathrm{C} & -0.36366900 & -0.05534400 & -1.04982000 \\ \mathrm{H} & -0.45321600 & 0.59610400 & -1.91326100 \\ \mathrm{C} & 1.80449700 & 0.10783400 & 0.05852700 \\ \mathrm{~N} & 0.81992800 & -0.22074300 & -0.57387400 \\ \mathrm{C} & 3.14838200 & -0.45003700 & 0.03344600 \\ \mathrm{H} & 5.04568400 & -2.81700000 & -1.48705100 \\ \mathrm{C} & 5.73606700 & -1.46603900 & 0.03552800 \\ \mathrm{C} & 4.11212900 & 0.08235400 & 0.88685000 \\ \mathrm{C} & 3.49358100 & -1.49895500 & -0.82435100 \\ \mathrm{C} & 4.78095400 & -2.00354400 & -0.82081400 \\ \mathrm{C} & 5.40254000 & -0.42396900 & 0.88821700 \\ \mathrm{H} & 3.84046000 & 0.89770700 & 1.54655200 \\ \mathrm{H} & 2.74216900 & -1.90963500 & -1.48889400 \\ \mathrm{C} & -2.55558100 & -2.45679800 & 0.83049700 \\ \mathrm{C} & -1.50484000 & -0.83502200 & -0.61709400 \\ \mathrm{C} & -3.79098700 & -2.25483600 & 0.22122800 \\ \mathrm{C} & -1.42837400 & -1.76263400 & 0.42387800 \\ \mathrm{C} & -2.74376900 & -0.62750200 & -1.23168700 \\ \mathrm{C} & -0.73992400 & 2.80459600 & -0.56766000 \\ \mathrm{O} & -0.14686500 & 3.18527000 & -1.54251800 \\ \mathrm{H} & 6.74540000 & -1.86289500 & 0.03509300 \\ \mathrm{H} & -0.47632600 & -1.94759300 & 0.90868100 \\ \mathrm{H} & -2.82435300 & 0.09636500 & -2.03545900 \\ \mathrm{H} & -4.81279900 & -1.14604600 & -1.30879700 \\ \mathrm{H} & -2.47247700 & -3.17708200 & 1.63807000 \\ \mathrm{H} & -3.86223900 & -1.32470700 & -0.81589700 \\ \mathrm{C} & -5.14866800 & -0.00501800 & 1.55349400 \\ \mathrm{H} & 1.79744200 & 2.61720200 & 0.24733800 \\ \mathrm{H} & -5.00828400 & -3.01151800 & 0.65439300 \\ \mathrm{H} & -5.78609297500 & -3.62187400 & -0.16269200 \\ \mathrm{H} & -3.67445600 & 1.49143400 \\ \mathrm{H} & & -2.33125500 & 0.96482900\end{array}$




\section{Product 5c-endo-TS}

$\begin{array}{lrrr}\mathrm{H} & 0.08786000 & 3.05795600 & -1.79602900 \\ \mathrm{C} & 1.10468200 & 2.18855200 & 0.84586600 \\ \mathrm{H} & 1.13362400 & 1.73130900 & 1.82727100 \\ \mathrm{C} & -0.10121600 & 2.45795400 & 0.29419100 \\ \mathrm{H} & -0.23024800 & 4.37436200 & -0.67067400 \\ \mathrm{C} & -1.95029500 & 3.08757400 & -1.00717400 \\ \mathrm{H} & -2.18672000 & 2.38167200 & -1.80845300 \\ \mathrm{H} & -2.51675700 & 4.00315700 & -1.17616800 \\ \mathrm{O} & -2.39308900 & 2.52511700 & 0.21931200 \\ \mathrm{C} & -0.44429200 & 3.32113800 & -0.87943600 \\ \mathrm{C} & -0.35510000 & -0.06944300 & -1.19125200 \\ \mathrm{H} & -0.46474000 & 0.45016400 & -2.13899700 \\ \mathrm{C} & 1.81989800 & 0.19810400 & -0.09514700 \\ \mathrm{~N} & 0.83756300 & -0.15066200 & -0.71874300 \\ \mathrm{C} & 3.14851800 & -0.39530800 & -0.05843300 \\ \mathrm{H} & 5.04960500 & -2.82590000 & -1.46996000 \\ \mathrm{C} & 5.70771100 & -1.47414400 & 0.06605800 \\ \mathrm{C} & 4.08821200 & 0.12411900 & 0.82939300 \\ \mathrm{C} & 3.50308200 & -1.46384400 & -0.88759500 \\ \mathrm{C} & 4.77729300 & -1.99741700 & -0.82561600 \\ \mathrm{C} & 5.36335500 & -0.41579200 & 0.89405600 \\ \mathrm{H} & 3.80715400 & 0.95082000 & 1.47093700 \\ \mathrm{H} & 2.76888700 & -1.86846100 & -1.57486900 \\ \mathrm{C} & -2.51784100 & -2.22808600 & 0.98427800 \\ \mathrm{C} & -1.47739700 & -0.83408200 & -0.68228800 \\ \mathrm{H} & -3.71273300 & -2.25582800 & 0.26409200 \\ \mathrm{C} & -1.41814200 & -1.53115100 & 0.52719000 \\ \mathrm{C} & -2.66826400 & -0.85836900 & -1.40661800 \\ \mathrm{C} & -1.34532500 & 2.05237200 & 0.94072900 \\ \mathrm{C} & -1.51065000 & 1.43636700 & 1.95752200 \\ \mathrm{O} & 6.70555100 & -1.89633300 & 0.11422100 \\ \mathrm{H} & -0.50830600 & -1.51179000 & 1.11455700 \\ \mathrm{H} & -2.73485000 & -0.32362700 & -2.34872800 \\ \mathrm{H} & -4.68361800 & -1.56282600 & -1.51794200 \\ \mathrm{H} & -2.45488500 & -2.75493000 & 1.93097300 \\ \mathrm{H} & -3.76646200 & -1.55934400 & -0.93820200 \\ \mathrm{H} & -.08891100 & -0.01050600 & 1.58987500 \\ \mathrm{H} & -00619700 & 2.67635300 & 0.49406700 \\ \mathrm{H} & -3.72162700 & -2.99608900 & 0.07113000\end{array}$




\section{Product 5d - exo-TS}

\begin{tabular}{|c|c|c|c|}
\hline $\mathrm{H}$ & 0.55903200 & 2.53577300 & 2.66367700 \\
\hline $\mathrm{C}$ & 2.88847800 & 1.78175100 & 1.14098200 \\
\hline $\mathrm{H}$ & 3.06036300 & 1.32724200 & 2.10877400 \\
\hline $\mathrm{C}$ & 1.68828300 & 2.32615200 & 0.83167800 \\
\hline $\mathrm{H}$ & -0.07766800 & 1.31610900 & 1.56183100 \\
\hline $\mathrm{C}$ & -0.42392200 & 3.36335600 & 0.89964400 \\
\hline $\mathrm{H}$ & -0.51710800 & 4.27323500 & 1.49692900 \\
\hline $\mathrm{H}$ & -1.42064200 & 3.01547800 & 0.63082100 \\
\hline $\mathrm{O}$ & 0.25344900 & 3.70569900 & -0.30694300 \\
\hline $\mathrm{C}$ & 0.41211700 & 2.29353500 & 1.60919400 \\
\hline $\mathrm{C}$ & 0.96284700 & 0.42668900 & -1.16378600 \\
\hline $\mathrm{H}$ & 1.16729500 & 1.15289200 & -1.94373100 \\
\hline $\mathrm{C}$ & 2.95816700 & -0.26783600 & 0.05598000 \\
\hline $\mathrm{N}$ & 1.98070300 & -0.17911400 & -0.65829100 \\
\hline $\mathrm{C}$ & 4.04538600 & -1.23316800 & 0.04758900 \\
\hline $\mathrm{H}$ & 5.22363500 & -3.94092500 & -1.62313900 \\
\hline $\mathrm{C}$ & 6.15580300 & -3.04122500 & 0.09194200 \\
\hline $\mathrm{C}$ & 5.03923600 & -1.13002600 & 1.01833100 \\
\hline $\mathrm{C}$ & 4.11858400 & -2.25244200 & -0.90700600 \\
\hline $\mathrm{C}$ & 5.16904100 & -3.15137700 & -0.88203700 \\
\hline $\mathrm{C}$ & 6.09162400 & -2.03162300 & 1.04109200 \\
\hline $\mathrm{H}$ & 4.97998900 & -0.33561100 & 1.75248800 \\
\hline $\mathrm{H}$ & 3.34501100 & -2.32839200 & -1.66240100 \\
\hline $\mathrm{C}$ & -2.04363800 & -1.26207900 & 0.31907200 \\
\hline $\mathrm{C}$ & -0.40246400 & 0.04540200 & -0.87646800 \\
\hline $\mathrm{C}$ & -3.09122300 & -0.57549700 & -0.29933000 \\
\hline $\mathrm{C}$ & -0.72442700 & -0.96127800 & 0.03923600 \\
\hline $\mathrm{C}$ & -1.44726400 & 0.73430300 & -1.49912300 \\
\hline $\mathrm{C}$ & 1.50072900 & 3.18159200 & -0.33767700 \\
\hline $\mathrm{O}$ & 2.26577700 & 3.43899200 & -1.22927100 \\
\hline $\mathrm{H}$ & 6.97892400 & -3.74722000 & 0.10779700 \\
\hline $\mathrm{H}$ & 0.06937100 & -1.52364200 & 0.51804900 \\
\hline $\mathrm{H}$ & -1.21736700 & 1.53029400 & -2.19913800 \\
\hline $\mathrm{H}$ & -3.55543400 & 0.99587800 & -1.69011100 \\
\hline $\mathrm{H}$ & -2.26826100 & -2.06747900 & 1.00942600 \\
\hline $\mathrm{C}$ & -2.76422400 & 0.42824200 & -1.21332500 \\
\hline $\mathrm{H}$ & 6.86273800 & -1.94622300 & 1.79794200 \\
\hline $\mathrm{H}$ & 3.75723300 & 2.06586300 & 0.55803200 \\
\hline $\mathrm{C}$ & -4.49728000 & -0.90255100 & 0.00124200 \\
\hline $\mathrm{C}$ & -4.89221900 & -1.24572000 & 1.29506900 \\
\hline $\mathrm{C}$ & -5.46848900 & -0.87519800 & -1.00057300 \\
\hline $\mathrm{C}$ & -6.21393600 & -1.55168700 & 1.57812400 \\
\hline $\mathrm{H}$ & -4.16034100 & -1.24780600 & 2.09518900 \\
\hline $\mathrm{C}$ & -6.79047600 & -1.18089200 & -0.71859200 \\
\hline $\mathrm{H}$ & -5.18025300 & -0.63684100 & -2.01835700 \\
\hline $\mathrm{C}$ & -7.16958900 & -1.52070700 & 0.57237900 \\
\hline $\mathrm{H}$ & -6.50079500 & -1.80714700 & 2.59237900 \\
\hline $\mathrm{H}$ & -7.52748000 & -1.16172100 & -1.51396400 \\
\hline $\mathrm{H}$ & -8.20383000 & -1.75977700 & 0.79343700 \\
\hline
\end{tabular}

- S33 - 


\section{Product 5d - endo-TS}

$\begin{array}{lrrr}\mathrm{H} & 2.48535900 & 3.26377000 & -1.51050000 \\ \mathrm{C} & 2.90446800 & 1.83456400 & 1.04837400 \\ \mathrm{H} & 2.68936600 & 1.30347200 & 1.96767400 \\ \mathrm{C} & 1.91158000 & 2.55113400 & 0.47186600 \\ \mathrm{H} & 2.49464400 & 4.49065100 & -0.24685400 \\ \mathrm{C} & 0.49638900 & 3.87869800 & -0.84556200 \\ \mathrm{H} & 0.12351700 & 3.35926600 & -1.73289200 \\ \mathrm{H} & 0.27043300 & 4.94002600 & -0.94490600 \\ \mathrm{O} & -0.21538200 & 3.38843500 & 0.28162500 \\ \mathrm{C} & 1.97826700 & 3.59240900 & -0.60101900 \\ \mathrm{C} & 0.98535200 & 0.40763300 & -1.34127100 \\ \mathrm{H} & 1.14893100 & 0.99656800 & -2.23927900 \\ \mathrm{C} & 3.00073600 & -0.17766900 & -0.07623200 \\ \mathrm{~N} & 2.03026100 & -0.11360700 & -0.80111700 \\ \mathrm{C} & 4.04332100 & -1.18833500 & 0.00551600 \\ \mathrm{H} & 5.18566800 & -4.00193600 & -1.50790700 \\ \mathrm{C} & 6.07043200 & -3.07771400 & 0.21917500 \\ \mathrm{C} & 4.99241000 & -1.08908300 & 1.02065400 \\ \mathrm{C} & 4.11808200 & -2.24590200 & -0.90615000 \\ \mathrm{C} & 5.12920400 & -3.18312400 & -0.79940100 \\ \mathrm{C} & 6.00105600 & -2.03353900 & 1.12980300 \\ \mathrm{H} & 4.92849200 & -0.26854600 & 1.72521600 \\ \mathrm{H} & 3.37586900 & -2.32234100 & -1.69246500 \\ \mathrm{C} & -1.97640200 & -1.00043400 & 0.47834200 \\ \mathrm{C} & -0.37109900 & 0.04237600 & -0.98469200 \\ \mathrm{C} & -3.03479900 & -0.57991100 & -0.32920800 \\ \mathrm{C} & -0.66769100 & -0.69854600 & 0.16090400 \\ \mathrm{H} & -1.42593400 & 0.46682500 & -1.79517100 \\ \mathrm{C} & -8.54942900 & 2.52553400 & 0.99583200 \\ \mathrm{C} & -108098000 & -1.90329500 & 1.91925700 \\ \mathrm{O} & 6.86071000 & -3.81587300 & 0.30161500 \\ \mathrm{H} & 0.13323600 & -1.03094300 & 0.80974000 \\ \mathrm{H} & -1.21704600 & 1.05200100 & -2.68516900 \\ \mathrm{H} & -3.53745800 & 0.52235800 & -2.10394600 \\ \mathrm{H} & -2.18357400 & -1.58682400 & 1.36609600 \\ \mathrm{H} & -2.73364500 & 0.15884200 & -1.47375300 \\ \mathrm{C} & 6.73403800 & -1.95446600 & 1.92429600 \\ \mathrm{H} & 3.94502400 & 2.01989200 & 0.80865500 \\ \mathrm{H} & -4.43048900 & -0.89817100 & 0.02394100 \\ \mathrm{C} & -4.85787600 & -0.84842900 & 1.35131400 \\ \mathrm{C} & -5.35680500 & -1.25093200 & -0.95806800 \\ \mathrm{C} & -6.17004900 & -1.14352900 & 1.68602400 \\ \mathrm{H} & -4.16112700 & -0.54571700 & 2.12502200 \\ \mathrm{H} & -6.66891200 & -1.54736800 & -0.62381800 \\ \mathrm{H} & -6.48482500 & -1.08873100 & 2.72245700 \\ \mathrm{H} & -1.31793100 & -1.99275100 \\ \mathrm{H} & -1.37136500 & -1.82824800 & -1.40106600 \\ \mathrm{H} & & & 0.96209300\end{array}$

- S34 - 


$\begin{array}{lrrr}\text { Product 5e }- \text { exo-TS } & & \\ \mathrm{H} & -1.30077200 & 2.27401900 & 2.54714900 \\ \mathrm{C} & 1.06036200 & 2.15352800 & 0.89724100 \\ \mathrm{H} & 1.43172200 & 1.93363300 & 1.89034300 \\ \mathrm{C} & -0.26924800 & 2.20508300 & 0.64704300 \\ \mathrm{H} & -1.52824500 & 0.76909500 & 1.65919500 \\ \mathrm{C} & -2.60299600 & 2.46270700 & 0.80639900 \\ \mathrm{H} & -2.96723200 & 3.36878400 & 1.29598500 \\ \mathrm{H} & -3.43433300 & 1.76833900 & 0.68950800 \\ \mathrm{O} & -2.15683200 & 2.82410700 & -0.49803000 \\ \mathrm{C} & -1.40951600 & 1.85116400 & 1.54642100 \\ \mathrm{C} & -0.38369200 & -0.08735200 & -1.03213700 \\ \mathrm{H} & -0.48194400 & 0.55704500 & -1.89971900 \\ \mathrm{C} & 1.78675500 & 0.11662000 & 0.06197900 \\ \mathrm{~N} & 0.80215200 & -0.23758400 & -0.55643700 \\ \mathrm{C} & 3.14149300 & -0.41181300 & 0.03347500 \\ \mathrm{H} & 5.09087300 & -2.71904400 & -1.51244600 \\ \mathrm{C} & 5.75040600 & -1.37043100 & 0.02582600 \\ \mathrm{C} & 4.09305400 & 0.13298500 & 0.89288000 \\ \mathrm{C} & 3.50966100 & -1.44359500 & -0.83565500 \\ \mathrm{C} & 4.80788700 & -1.91928600 & -0.83726500 \\ \mathrm{C} & 5.39393500 & -0.34548000 & 0.88996500 \\ \mathrm{H} & 3.80376000 & 0.93542100 & 1.56082700 \\ \mathrm{H} & 2.76783800 & -1.86356200 & -1.50515200 \\ \mathrm{C} & -2.52953800 & -2.51303300 & 0.87360200 \\ \mathrm{C} & -1.51566800 & -0.87787200 & -0.59364800 \\ \mathrm{C} & -3.73872300 & -2.29222700 & 0.24217300 \\ \mathrm{C} & -1.42022500 & -1.80194100 & 0.45181600 \\ \mathrm{C} & -2.75674300 & -0.68352300 & -1.20685300 \\ \mathrm{C} & -0.80765400 & 2.76115800 & -0.59154500 \\ \mathrm{O} & -0.23209400 & 3.12767300 & -1.58198100 \\ \mathrm{H} & 6.76822300 & -1.74490500 & 0.02155600 \\ \mathrm{H} & -0.46280700 & -1.97159900 & 0.93080300 \\ \mathrm{H} & -2.84625500 & 0.03718500 & -2.01201300 \\ \mathrm{H} & -4.83996000 & -1.24403000 & -1.26081700 \\ \mathrm{H} & -2.46791100 & -3.23542500 & 1.67827700 \\ \mathrm{H} & -3.87395900 & -1.38734500 & -0.79254100 \\ & 6.13047000 & 0.08217200 & 1.56031600 \\ \mathrm{H} & 1.74234800 & 2.62768800 & 0.20057300 \\ \mathrm{H} & & & \\ & -41366400 & -2.98022800 & 0.64619100\end{array}$




$\begin{array}{lrrr}\text { Product 5e }- \text { endo-TS } & & \\ \mathrm{H} & & & \\ \mathrm{C} & 0.02159200 & 3.05835800 & -1.80029000 \\ \mathrm{H} & 1.05719300 & 2.19787000 & 0.83803500 \\ \mathrm{C} & 1.09513200 & 1.73682400 & 1.81734700 \\ \mathrm{H} & -0.15336800 & 2.44559800 & 0.28684100 \\ \mathrm{C} & -0.31651800 & 4.36366000 & -0.66830900 \\ \mathrm{H} & -2.01609600 & 3.05040100 & -1.00886600 \\ \mathrm{H} & -2.24347500 & 2.35453600 & -1.82152900 \\ \mathrm{O} & -2.59866700 & 3.95873800 & -1.16091300 \\ \mathrm{C} & -2.44607300 & 2.45949900 & 0.20909300 \\ \mathrm{C} & -0.51366500 & 3.30830100 & -0.88182800 \\ \mathrm{H} & -0.38010400 & -0.08663900 & -1.18002600 \\ \mathrm{C} & -0.50329900 & 0.42067300 & -2.13251200 \\ \mathrm{~N} & 1.80029500 & 0.21431800 & -0.10423200 \\ \mathrm{C} & 0.81733900 & -0.15070800 & -0.71616300 \\ \mathrm{H} & 3.13784000 & -0.35630900 & -0.06786900 \\ \mathrm{C} & 5.07317200 & -2.76240200 & -1.47439600 \\ \mathrm{C} & 5.71356000 & -1.39378600 & 0.05418200 \\ \mathrm{C} & 4.07143800 & 0.18231800 & 0.81505700 \\ \mathrm{C} & 3.50673200 & -1.42282500 & -0.89355400 \\ \mathrm{C} & 4.78938000 & -1.93566700 & -0.83284500 \\ \mathrm{H} & 5.35490000 & -0.33739100 & 0.87865800 \\ \mathrm{H} & 3.77939500 & 1.00746800 & 1.45363200 \\ \mathrm{C} & 2.77738000 & -1.84206600 & -1.57718300 \\ \mathrm{C} & -2.49034600 & -2.24480900 & 1.05234200 \\ \mathrm{C} & -1.48785700 & -0.86304600 & -0.65507900 \\ \mathrm{C} & -3.64842900 & -2.28823300 & 0.30021200 \\ \mathrm{C} & -1.41184800 & -1.52790200 & 0.57104800 \\ \mathrm{C} & -2.67750400 & -0.92552500 & -1.38292500 \\ \mathrm{O} & -1.38852600 & 2.00506000 & 0.92695000 \\ \mathrm{H} & -1.53988400 & 1.37149900 & 1.93538000 \\ \mathrm{H} & 6.71804100 & -1.79995700 & 0.10149300 \\ \mathrm{H} & -0.50418200 & -1.46985700 & 1.15835200 \\ \mathrm{H} & -2.75220600 & -0.41030600 & -2.33470700 \\ \mathrm{H} & -4.69372400 & -1.69295800 & -1.46676300 \\ \mathrm{C} & -2.44926500 & -2.75630800 & 2.00571600 \\ & -3.76560200 & -1.63790900 & -0.91181900 \\ \mathrm{H} & 6.07583800 & 0.08218300 & 1.57073400 \\ \mathrm{H} & 1.94939600 & 2.70334500 & 0.48748300 \\ \mathrm{H} & -49447600 & -2.98310100 & 0.76372400\end{array}$




\section{Product 5f - exo-TS}

$\begin{array}{lrrr}\mathrm{H} & -0.04785000 & 2.58765700 & -2.59776500 \\ \mathrm{C} & -2.37941400 & 1.96621800 & -1.02678500 \\ \mathrm{H} & -2.62243000 & 1.58955300 & -2.01255400 \\ \mathrm{C} & -1.12535600 & 2.38431100 & -0.73311700 \\ \mathrm{H} & 0.52753300 & 1.27393300 & -1.57377800 \\ \mathrm{C} & 1.05732800 & 3.25630000 & -0.83896500 \\ \mathrm{H} & 1.18188600 & 4.19168100 & -1.38933800 \\ \mathrm{H} & 2.04063700 & 2.83048700 & -0.64290600 \\ \mathrm{O} & 0.46408800 & 3.56931200 & 0.41895400 \\ \mathrm{C} & 0.11570800 & 2.28783100 & -1.56085300 \\ \mathrm{C} & -0.51537400 & 0.33475100 & 1.12566800 \\ \mathrm{H} & -0.63769600 & 1.03527500 & 1.94501800 \\ \mathrm{C} & -2.59749700 & -0.12246900 & -0.05694000 \\ \mathrm{~N} & -1.59290400 & -0.16094200 & 0.62260500 \\ \mathrm{C} & -3.77155000 & -0.97869500 & -0.05389800 \\ \mathrm{H} & -5.19293700 & -3.56837000 & 1.61471200 \\ \mathrm{C} & -6.04183400 & -2.58036000 & -0.09500200 \\ \mathrm{C} & -4.75543900 & -0.77768800 & -1.01951400 \\ \mathrm{C} & -3.93620600 & -1.99008900 & 0.89772800 \\ \mathrm{C} & -5.06688300 & -2.78539900 & 0.87542100 \\ \mathrm{C} & -5.88665700 & -1.57819400 & -1.04165300 \\ \mathrm{H} & -4.62586900 & 0.01132400 & -1.75042700 \\ \mathrm{H} & -3.17021000 & -2.14188300 & 1.64941300 \\ \mathrm{C} & 2.29086600 & -1.54384000 & -0.51895500 \\ \mathrm{C} & 0.80485300 & -0.14941600 & 0.78518100 \\ \mathrm{C} & 3.37815300 & -0.96506700 & 0.11932600 \\ \mathrm{C} & 1.01217800 & -1.13475200 & -0.18539800 \\ \mathrm{C} & 1.91826700 & 0.41504000 & 1.41437700 \\ \mathrm{C} & -0.82231000 & 3.15235600 & 0.47273300 \\ \mathrm{O} & -1.52847500 & 3.41983000 & 1.40864100 \\ \mathrm{H} & -6.92772200 & -3.20578700 & -0.11007000 \\ \mathrm{H} & 0.16089700 & -1.59301000 & -0.67557600 \\ \mathrm{H} & 1.77505400 & 1.18638200 & 2.16297100 \\ \mathrm{H} & 4.05800100 & 0.45656100 & 1.57713200 \\ \mathrm{H} & 2.44518700 & -2.31046700 & -1.26789500 \\ \mathrm{C} & 3.20092000 & 0.01377000 & 1.08566400 \\ \mathrm{H} & -6.64891500 & -1.41869000 & -1.79545000 \\ \mathrm{H} & -3.20007800 & 2.29190900 & -0.39758200 \\ \mathrm{Br} & 5.12204100 & -1.52087900 & -0.33100000\end{array}$




\section{Product 5f - endo-TS}

$\begin{array}{lrrr}\mathrm{H} & -1.78935900 & 3.30749300 & 1.60055200 \\ \mathrm{C} & -2.39915500 & 1.99922700 & -0.98754100 \\ \mathrm{H} & -2.25349700 & 1.47854200 & -1.92617800 \\ \mathrm{C} & -1.33437700 & 2.60920500 & -0.41694100 \\ \mathrm{H} & -1.74812100 & 4.56520000 & 0.36923500 \\ \mathrm{C} & 0.21827700 & 3.79382900 & 0.88568700 \\ \mathrm{H} & 0.57691400 & 3.24192800 & 1.75915900 \\ \mathrm{H} & 0.52589100 & 4.83441600 & 0.98531100 \\ \mathrm{O} & 0.85985100 & 3.26112800 & -0.26419100 \\ \mathrm{C} & -1.28796300 & 3.62285000 & 0.68315900 \\ \mathrm{C} & -0.52365500 & 0.36689800 & 1.27181000 \\ \mathrm{H} & -0.60493700 & 0.95488000 & 2.18124900 \\ \mathrm{C} & -2.62967800 & -0.03341400 & 0.08904200 \\ \mathrm{~N} & -1.62631700 & -0.06651800 & 0.76825600 \\ \mathrm{C} & -3.76220300 & -0.94270700 & 0.03634800 \\ \mathrm{H} & -5.09624400 & -3.65412300 & 1.57894000 \\ \mathrm{C} & -5.95296800 & -2.64516100 & -0.11454600 \\ \mathrm{C} & -4.73244200 & -0.75293500 & -0.94545600 \\ \mathrm{C} & -3.89908300 & -1.99513800 & 0.94683800 \\ \mathrm{C} & -4.99160000 & -2.83909900 & 0.87162900 \\ \mathrm{C} & -5.82279700 & -1.60518700 & -1.02344100 \\ \mathrm{H} & -4.62053700 & 0.06359200 & -1.64871700 \\ \mathrm{H} & -3.14055500 & -2.14191800 & 1.70715000 \\ \mathrm{C} & 2.23290300 & -1.28309500 & -0.67023800 \\ \mathrm{C} & 0.78362800 & -0.10848100 & 0.86220100 \\ \mathrm{C} & 3.32181600 & -0.95186200 & 0.12224500 \\ \mathrm{C} & 0.97066800 & -0.86010900 & -0.29966300 \\ \mathrm{C} & 1.89790000 & 0.21756500 & 1.63750800 \\ \mathrm{C} & 0.00835000 & 2.48209800 & -0.97626500 \\ \mathrm{O} & 0.38083200 & 1.84350000 & -1.92185200 \\ \mathrm{H} & -6.80731600 & -3.31060100 & -0.17317000 \\ \mathrm{H} & 0.12240100 & -1.10330900 & -0.92712400 \\ \mathrm{H} & 1.77156800 & 0.80395300 & 2.54153900 \\ \mathrm{H} & 4.02527600 & 0.05600900 & 1.88193100 \\ \mathrm{H} & 2.37410200 & -1.85789400 & -1.57642100 \\ \mathrm{C} & 3.16582900 & -0.19914400 & 1.27527500 \\ \mathrm{H} & -6.57216100 & -1.45719900 & -1.79237300 \\ \mathrm{H} & -3.41354600 & 2.26510500 & -0.71375500 \\ \mathrm{Br} & 5.04366200 & -1.53341500 & -0.37779000\end{array}$




\section{Product 5g - exo-TS}

$\begin{array}{lrrr}\mathrm{H} & 0.78800200 & 2.41614300 & -2.57729600 \\ \mathrm{C} & -1.57441700 & 2.07550900 & -0.96363700 \\ \mathrm{H} & -1.89021800 & 1.76549000 & -1.95201700 \\ \mathrm{C} & -0.26778700 & 2.30532800 & -0.69267800 \\ \mathrm{H} & 1.18813200 & 0.99795300 & -1.61087300 \\ \mathrm{C} & 2.01583800 & 2.85625500 & -0.82785100 \\ \mathrm{H} & 2.26751600 & 3.77958400 & -1.35459000 \\ \mathrm{H} & 2.92872800 & 2.28425100 & -0.66628800 \\ \mathrm{O} & 1.49905400 & 3.21434100 & 0.45152600 \\ \mathrm{C} & 0.92825800 & 2.05968800 & -1.55496600 \\ \mathrm{C} & 0.08891000 & 0.12465400 & 1.09529600 \\ \mathrm{H} & 0.08484000 & 0.80707400 & 1.93877800 \\ \mathrm{C} & -2.06256500 & 0.00793000 & -0.04642000 \\ \mathrm{~N} & -1.05870300 & -0.19416500 & 0.60501900 \\ \mathrm{C} & -3.34418400 & -0.67724200 & -0.04035200 \\ \mathrm{H} & -5.03959800 & -3.17853800 & 1.50265200 \\ \mathrm{C} & -5.81862400 & -1.94101800 & -0.07264100 \\ \mathrm{C} & -4.33257600 & -0.26357800 & -0.93082900 \\ \mathrm{C} & -3.60654600 & -1.73257900 & 0.83892900 \\ \mathrm{C} & -4.83816300 & -2.36056500 & 0.82014100 \\ \mathrm{C} & -5.56684900 & -0.89389200 & -0.94701300 \\ \mathrm{H} & -4.12544700 & 0.55713800 & -1.60688800 \\ \mathrm{H} & -2.83604900 & -2.05023900 & 1.53187000 \\ \mathrm{C} & 2.56306800 & -2.06910600 & -0.68617600 \\ \mathrm{C} & 1.31916800 & -0.52900500 & 0.70448700 \\ \mathrm{C} & 3.73433200 & -1.67616900 & -0.05583700 \\ \mathrm{C} & 1.36364300 & -1.49527000 & -0.30562000 \\ \mathrm{C} & 2.51494500 & -0.15356700 & 1.32384500 \\ \mathrm{C} & 0.16768600 & 2.98287500 & 0.52644100 \\ \mathrm{O} & -0.47277400 & 3.31785000 & 1.48757600 \\ \mathrm{H} & -6.78428600 & -2.43452100 & -0.08406100 \\ \mathrm{H} & 0.44586400 & -1.80752900 & -0.79037200 \\ \mathrm{H} & 2.49896600 & 0.59996900 & 2.10333900 \\ \mathrm{H} & 4.64384100 & -0.42649200 & 1.42993200 \\ \mathrm{H} & 2.59528800 & -2.82061900 & -1.46512500 \\ \mathrm{C} & 3.71965600 & -0.72021100 & 0.94827300 \\ \mathrm{H} & -6.33313200 & -0.56763000 & -1.64056500 \\ \mathrm{H} & -2.32674000 & 2.49356600 & -0.30430600 \\ \mathrm{Cl} & 5.23954200 & -2.39495200 & -0.52955700\end{array}$




\section{Product 5g - endo-TS}

$\begin{array}{lrrr}\mathrm{H} & -0.77119800 & 3.23717700 & 1.68048100 \\ \mathrm{C} & -1.58824700 & 2.10942200 & -0.92924700 \\ \mathrm{H} & -1.53261200 & 1.60226500 & -1.88478100 \\ \mathrm{C} & -0.43992200 & 2.54864500 & -0.36408500 \\ \mathrm{H} & -0.55211000 & 4.51663100 & 0.49022400 \\ \mathrm{C} & 1.28062700 & 3.44393900 & 0.95591200 \\ \mathrm{H} & 1.55921700 & 2.80247900 & 1.79709200 \\ \mathrm{H} & 1.74027300 & 4.42137000 & 1.09966000 \\ \mathrm{O} & 1.82608600 & 2.87871600 & -0.22747100 \\ \mathrm{C} & -0.23546600 & 3.50607300 & 0.76772400 \\ \mathrm{C} & 0.08789800 & 0.14765600 & 1.23027800 \\ \mathrm{H} & 0.11613200 & 0.70885400 & 2.15957900 \\ \mathrm{C} & -2.08212700 & 0.09691600 & 0.09665600 \\ \mathrm{~N} & -1.07811100 & -0.10299200 & 0.74601800 \\ \mathrm{C} & -3.33598500 & -0.63682700 & 0.04972300 \\ \mathrm{H} & -5.00262000 & -3.19972100 & 1.52132300 \\ \mathrm{C} & -5.76016200 & -1.98830000 & -0.08443000 \\ \mathrm{C} & -4.30129400 & -0.25695900 & -0.88050200 \\ \mathrm{C} & -3.59512400 & -1.70394800 & 0.91552100 \\ \mathrm{C} & -4.80316600 & -2.37320700 & 0.84855400 \\ \mathrm{C} & -5.50913300 & -0.93336100 & -0.94959100 \\ \mathrm{H} & -4.09388200 & 0.56871600 & -1.55057600 \\ \mathrm{H} & -2.84031200 & -1.99930800 & 1.63519800 \\ \mathrm{C} & 2.52167000 & -1.81920000 & -0.84300500 \\ \mathrm{C} & 1.30143800 & -0.49616600 & 0.76620300 \\ \mathrm{C} & 3.66859100 & -1.67211900 & -0.07788700 \\ \mathrm{C} & 1.34537300 & -1.23080500 & -0.42064200 \\ \mathrm{C} & 2.47287600 & -0.35746000 & 1.51285800 \\ \mathrm{C} & 0.86372900 & 2.25527000 & -0.95247100 \\ \mathrm{O} & 1.13053400 & 1.60861800 & -1.92776400 \\ \mathrm{H} & -6.70592800 & -2.51653500 & -0.13610100 \\ \mathrm{H} & 0.45310500 & -1.33163400 & -1.02567400 \\ \mathrm{H} & 2.45863100 & 0.21381200 & 2.43504900 \\ \mathrm{H} & 4.56296000 & -0.83193300 & 1.67982800 \\ \mathrm{H} & 2.55732500 & -2.38132900 & -1.76731900 \\ \mathrm{C} & 3.65614900 & -0.94059200 & 1.09868000 \\ \mathrm{H} & -6.25530600 & -0.63695900 & -1.67762500 \\ \mathrm{H} & -2.55097600 & 2.50427000 & -0.62626700 \\ \mathrm{Cl} & 5.14498600 & -2.41469200 & -0.60304900\end{array}$


$\Delta E$ and $\Delta G$ energies, entropy $(\Delta S)$, and imaginary frequencies ( $\left.\nu_{\text {img }}\right)$ of transition states optimized by MPW1K/aug-cc-pVDZ method.

\begin{tabular}{|c|c|c|c|c|c|}
\hline \multicolumn{2}{|c|}{ Structure } & & & $\Delta S$ & $v_{i m g}$ \\
\hline \multirow{2}{*}{$5 \mathbf{a}$} & exo-TS & -939.200183 & -938.930345 & 146.546 & -204.4032 \\
\hline & endo-TS & -939.195461 & -938.925880 & 146.513 & -190.7919 \\
\hline \multirow{2}{*}{$5 b$} & exo-TS & -1143.654118 & -1143.385175 & 160.568 & -235.3105 \\
\hline & endo-TS & -1143.650102 & -1143.381527 & 160.823 & -213.4821 \\
\hline \multirow{2}{*}{$5 c$} & exo-TS & -978.514107 & -978.219135 & 156.186 & -198.0588 \\
\hline & endo-TS & -978.509536 & -978.215099 & 156.725 & -183.1026 \\
\hline \multirow{2}{*}{$5 d$} & exo-TS & -1170.220738 & -1169.874705 & 170.203 & -203.2897 \\
\hline & endo-TS & -1170.216275 & -1169.870906 & 171.007 & -190.0593 \\
\hline \multirow{2}{*}{$5 e$} & exo-TS & -1038.416281 & -1038.156008 & 150.881 & -200.0925 \\
\hline & endo-TS & -1038.412120 & -1038.152204 & 151.140 & -182.9571 \\
\hline \multirow{2}{*}{$5 f$} & exo-TS & -3512.901050 & -3512.644668 & 156.186 & -209.4096 \\
\hline & endo-TS & -3512.896682 & -3512.640852 & 156.791 & -192.1888 \\
\hline \multirow{2}{*}{$5 g$} & exo-TS & -1398.828304 & -1398.570696 & 154.341 & -207.5180 \\
\hline & endo-TS & -1398.823953 & -1398.566537 & 154.221 & -190.5400 \\
\hline
\end{tabular}

